Solar Physics

DOI: $10.1007 / \bullet \bullet \bullet \bullet \bullet-\bullet \bullet \bullet-\bullet \bullet \bullet-\bullet \bullet \bullet \bullet-\bullet$

\title{
Coronal Shock Waves, EUV waves, and Their Relation to CMEs. I. Reconciliation of "EIT waves", Type II Radio Bursts, and Leading Edges of CMEs
}

\author{
V.V. Grechnev ${ }^{1}$ - A.M. Uralov ${ }^{1}$. \\ I.M. Chertok ${ }^{2}$ - I.V. Kuzmenko ${ }^{3}$. \\ A.N. Afanasyev ${ }^{1}$. N.S. Meshalkina ${ }^{1}$. \\ S.S. Kalashnikov ${ }^{1}$. Y. Kubo ${ }^{4}$
}

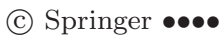

\begin{abstract}
We show examples of excitation of coronal waves by flare-related abrupt eruptions of magnetic rope structures. The waves presumably rapidly steepened into shocks and freely propagated afterwards like decelerating blast waves that showed up as Moreton waves and EUV waves. We propose a simple quantitative description for such shock waves to reconcile their observed propagation with drift rates of metric type II bursts and kinematics of leading edges of coronal mass ejections (CMEs). Taking account of different plasma density falloffs for propagation of a wave up and along the solar surface, we demonstrate a close correspondence between drift rates of type II bursts and speeds of EUV waves, Moreton waves, and CMEs observed in a few known events.
\end{abstract}

Keywords: Coronal Mass Ejections, Low Coronal Signatures; Coronal Mass Ejections, Initiation and Propagation; Prominences, Active; Radio Bursts, Dynamic Spectrum; Radio Bursts, Type II; Waves, Shock

\section{Introduction}

Some solar eruptions are accompanied by large-scale wave-like disturbances visible in various spectral ranges. Moreton waves (Moreton, 1960) observed in the $\mathrm{H} \alpha$ line have been initially proposed by Uchida (1968) to be a chromospheric trail of a coronal fast-mode MHD wave. Observations of the low corona in extreme

\footnotetext{
${ }^{1}$ Institute of Solar-Terrestrial Physics SB RAS, Lermontov St. 126A, Irkutsk 664033, Russia email: grechnev@iszf.irk.ru ${ }^{2}$ Pushkov Institute of Terrestrial Magnetism, Ionosphere and Radio Wave Propagation (IZMIRAN), Troitsk, Moscow Region, 142190 Russia email: ichertok@izmiran.ru

${ }^{3}$ Ussuriysk Astrophysical Observatory, Solnechnaya St. 21, Primorsky Krai, Gornotaezhnoe 692533, Russia email: kuzmenko_irina@mail.ru

${ }^{4}$ National Institute of Information and Communications

Technology, Tokyo, Japan email: kubo@nict.go.jp
} 
ultraviolet (EUV) with EIT (Delaboudinière et al., 1995) on SOHO revealed large-scale wave-like disturbances visible as fronts of enhanced (but still low) brightness, either quasi-stationary or propagating over large distances up to the whole disk along the solar surface or expanding above the limb. These transients called "EIT waves" (or "EUV waves") are registered by a number of EUV telescopes - EIT, TRACE, STEREO/EUVI, SDO/AIA, etc. (see Warmuth, 2007; Wills-Davey and Attrill, 2009; Gallagher and Long, 2010 for a review).

Analysis of observations and interpretation of such phenomena meet problems. Glaring flare emission hampers detection of faint EUV waves. Many data are limited by 12-min imaging rate of EIT. Faster TRACE observations (Handy et al., 1999) have a small field of view. It is often difficult to reliably identify and trace a moving feature of interest. To overcome these difficulties, special methods are employed, but they might contribute artifacts. Multi-instrument analyses sometimes encounter timing problems. Thus, some results used in interpretation and modeling might not be completely adequate to the observed phenomena.

The nature of EUV waves has been debated starting from their discovery (Thompson et al., 1998). The most popular interpretations of a near-surface EUV wave are $i$ ) traces of an MHD fast-mode wave in the lower corona (e.g., Thompson et al., 1999; Warmuth et al. 2001, 2004b; Khan and Aurass, 2002; Hudson and Warmuth, 2004) and ii) plasma compression in bases of coronal loops in their successive stretching by an expanding CME (e.g., Delannée, 2000). A numerical 2D MHD simulation of a magnetic flux rope eruption (Chen, Fang, and Shibata, 2005) revealed both the $(i)$ and (ii) disturbances. Schmidt and Ofman (2010) presented the first 3D MHD modeling of an "EIT wave" as a disturbance produced by an eruption-driven shock wave. A fast-mode wave detected in the simulation corresponded to an EUV wave observed in the modeled event including reflection from a coronal hole in support of the wave hypothesis.

None of existing models describes all properties of EUV waves. Most likely, this is because the multitude of transients observed as EUV waves actually correspond to different phenomena. This conjecture is supported by a variety of morphologic and dynamic characteristics of observed EUV waves. For example, 1) their velocities estimated from observations of some events exceeded the coronal fast-mode speed, whereas they were lower in other events (Wills-Davey, DeForest, and Stenflo, 2007; Warmuth, 2010); 2) the wave front can be either diffuse or sharp; 3) kinematics of an EUV wave can be incompatible with the fast-mode MHD wave model (Zhukov, Rodriguez, and de Patoul, 2009). On the other hand, such properties of EUV waves as deceleration, decay, and broadening the disturbance (Warmuth et al. 2001, 2004a, 2004b; Long et al., 2008; Veronig et al., 2010), bypassing regions of an increased Alfvén velocity - coronal holes and active regions (Thompson et al., 1999), possible reflections (Veronig, Temmer, and Vršnak, 2008; Gopalswamy et al., 2009) appear to correspond to the hypothesis of a coronal MHD wave. Considerations of wave-like transients sometimes observed in EUV to expand above the limb also suggest that different phenomena might be involved (see, e.g., Zhukov and Auchère, 2004; Grechnev et al., 2006b, 2008; Pohjolainen, Hori, and Sakurai, 2008; Meshalkina et al., 2009; Chertok, Grechnev, and Uralov, 2009; Cohen et al., 2009). In paper III (Grechnev 
et al., 2011) we consider an event with a two-component "EIT wave"; a propagating component matched properties of a coronal shock wave, while a stationary component was presumably associated with a stretched CME structure.

If some EUV waves are really due to coronal shock waves, then their correspondence with signatures of shocks in higher corona is expected. Type II radio bursts are considered as manifestations of shock waves propagating upwards in the corona (e.g., Vršnak and Cliver, 2008). Klassen et al. (2000) concluded that almost all metric type II bursts were accompanied by EUV waves but stated no correlation between their speeds; on average, the speeds of EUV waves were three times less than estimates from drift rates of type II bursts. Biesecker et al. (2002) found that many EUV waves were not associated with type II bursts.

Sheeley, Hakala, and Wang (2000) proposed that kinks or deflections of coronal rays at the flanks and rear ends of fast CMEs could be signatures of shock waves. An important common property of these flank/rear kinks was their deceleration, while the authors did not reveal deceleration of CME leading edges ${ }^{1}$. A usually considered scenario for the formation of shock waves, which might show up in LASCO images, seems to be incompatible with wave signatures on the solar surface. In this scenario, when the CME speed exceeds the local fast-mode speed at heliocentric distances $\gtrsim 1.5 R_{\odot}$, a bow shock forms continuously pressed by a fast CME. However, a bow shock followed by a Mach cone can only be formed by a supersonic body of a fixed size, whereas CMEs expand omnidirectionally (with respect to their center). Hence, the conic bow shock geometry appears to be unlikely for wide CMEs. Since neither mechanisms nor heights of the shock formation have been established, possible association between surface EUV waves and CME components cannot be excluded. Indeed, Veronig et al. (2010) found the upper part of an expanding EUV dome to coincide with a white-light CME, while the lower skirt of the dome was a surface EUV wave.

For these reasons it is difficult to expect that all observed properties of EUV waves could be explained by a single mechanism. Based on this assumption, our three companion papers are focused on those EUV waves, which are most likely associated with coronal shock waves. We address a few events, all of which were previously studied. Even for a rather uniform subset of phenomena probably corresponding to similar processes, seemingly contradictions between some observational facts might occur. We endeavor to reconcile such discrepancies.

One of challenging issues is reconciliation of EUV wave velocities and drift rates of type II bursts. This is a subject of the present paper I, the first one of the three companion papers. Assuming the shock wave nature of EUV waves under consideration, we try to settle disagreement between different studies. In this paper we use a simplest approximation of a self-similar shock wave that is convenient in comparisons with observations, which often show self-similarity of the wave front expansion. However, this approximation corresponds to the strong shock wave limit and cannot apply to all stages of events. A weaker shock seems to be more realistic, but its calculations are more complex. Propagation of a weak

\footnotetext{
${ }^{1}$ The SOHO LASCO CME Catalog at http://cdaw.gsfc.nasa.gov/CME_list/ (afterwards 'the CME Catalog'; Yashiro et al., 2004) shows pronounced decelerations for three of the four events considered by the authors.
} 
shock along the solar surface is analytically modeled in Paper II (Afanasyev and Uralov, 2011). Paper III analyzes propagation of a probable shock wave in the 17 January 2010 event using both strong and weak shock approximations.

\section{Methodical Issues}

Excitation of shock waves in eruptive events seems to be undoubted, but their sources have not been established. Three possible exciters of shock waves are considered: $i$ ) a pressure pulse produced by a flare, $i i$ ) a super-Alfvénic piston, and iii) an impulsive piston. In case (ii), a bow shock continuously driven by a supersonic CME is expected to appear with kinematics determined by the driver. In case $(i)$ or $(i i i)$, the shock wave expelled by an impulsive driver propagates afterwards freely like a decelerating blast wave (cf. Pomoell, Vainio, and Kissmann, 2008). Observations suggest that shock waves excited by impulsive drivers and freely propagating in the low corona do exist. This is indicated by deceleration of Moreton/EUV waves (e.g., Warmuth et al. 2001, 2004a), differences between propagation directions of the wave and a possible driver (Hudson et al., 2003), very early appearance of type II bursts. We accept this possibility as a working hypothesis and use a description of propagation of a blast shock wave. It is possible to calculate it analytically for two limits. One limit is a strong selfsimilar wave, whose length along a propagation direction is comparable with the curvature radius of the wave front. The opposite limit is a weak shock wave, whose length is much less than the curvature radius of the front and a typical size of inhomogeneities in the medium. Grechnev et al. (2008) found that a formal usage of expressions for propagation of a strong self-similar shock wave excited by a point-like explosion in a gas allowed to fit the speeds and positions of a Moreton wave as well as an EUV wave at the initial stage of the motion.

\subsection{Self-Similar Shock Wave Approximation}

Grechnev et al. (2008) used a simple model to describe propagation of such a blast-like wave in plasma with a radial power-law (PL) density falloff $\delta$ from an eruption center, $n=n_{0}\left(x / h_{0}\right)^{-\delta}$ with $x$ being the distance and $n_{0}$ the density at a distance of $h_{0}$. We use $h_{0} \approx 100 \mathrm{Mm}$, close to the scale height. (Our notations are different from those used in the papers listed above). Self-similarity of a shock wave is ensured by a presumable large pressure excess inside the volume confined by the shock front over a non-disturbed medium. Propagation of a shock wave in the self-similar approximation is determined by plasma density distribution being almost insensitive to the magnetic fields. Such a wave decelerates when $\delta<3$ due to a growing mass of swept-up material. Propagation of a strong shock vs. time $t$ in plasma with a PL density model is described by an expression

$$
x(t) \propto t^{2 /(5-\delta)}
$$

The approximation has a singularity at $x \rightarrow 0$ (here also the wave velocity $v \rightarrow \infty$ ); however, wave signatures are not observed at small distances. The approximation becomes inaccurate at large distances, being not limited from 
below by the fast-mode speed. A wave traveling along the solar surface weakens at large distances and propagates, in the first approximation, in a flat-layered atmosphere. Expression (1) describes strong spherical shock waves, which seem to be unrealistic in solar conditions, but its usage within some range of distances can be justified. 1) An enhanced plasma density above an active region falls off both vertically and horizontally. A power-law description of the falloff seems to be acceptable. 2) The self-similar solution of a strong wave satisfactorily describes damping of a gas-dynamic shock wave up to Mach numbers $M \approx 2$, when the wave is neither strong nor weak. 3) Applicability of gas-dynamic selfsimilar solutions to MHD blast shock waves is not obvious, because account of the magnetic field seems to be necessary. We note the following. With $M \gg 2$, the gas pressure behind the shock front exceeds the magnetic pressure, even if $\beta=C_{\mathrm{S}}^{2} / V_{\mathrm{A}}^{2} \ll 1$ in non-disturbed plasma before the shock front; here $M$ is a ratio of the shock speed to the fast-mode speed before the front, $C_{\mathrm{S}}$ and $V_{\mathrm{A}}$ are the sound and Alfvén speeds. That is, the plasma flow behind the shock front has a gas-dynamic character. The role of magnetic fields is also not crucial for mediumintensity shocks $(M \geq 2)$, which also strongly heat plasma, thus significantly increasing its pressure. For example, with $M \approx 2$, the plasma pressure behind the front of a wave perpendicular to the magnetic field is equal to the magnetic pressure before the front, even if $\beta \ll 1$ there. For a switch-on shock wave running along the magnetic field this occurs with a Mach number $M \approx 1.5$.

Expression (1) was obtained under an assumption that the $\delta$ index was independent of the wave propagation direction $\vartheta$. We will formally use this expression also when $\delta=\delta(\vartheta)$, if variations of $\delta$ are small with the change of the direction, i.e., $\pi d \delta \ll d \vartheta$. Note that in a limit of a weak, short shock wave, its propagation is determined by a local value of $\delta$ even if this condition is not satisfied. The above considerations lead to a heuristic conclusion about a possibility to use expression (1) for approximate estimates of kinematic characteristics of shock waves of intermediate intensity propagating in medium with $\delta=\delta(\vartheta)$.

It is useful to compare the power-law coronal density model with other popular models. The Newkirk (1961) model $\left(n_{e}=4.2 \times 10^{4} \times 10^{4.32 / r}, r\right.$ is the heliocentric distance expressed in solar radii) describes the radial plasma density distribution in a coronal streamer. The Saito model (Saito, 1970) describes the density distribution above the quiet Sun depending on the latitude $\phi$

$$
\frac{n_{e}(r, \phi)}{10^{8}}=\frac{3.09}{r^{16}}(1-0.5 \sin \phi)+\frac{1.58}{r^{6}}(1-0.95 \sin \phi)+\frac{0.0251}{r^{2.5}}\left(1-\sin ^{0.5} \phi\right)
$$

Figure 1a presents the Saito model for $\phi=0^{\circ}, 60^{\circ}$, and the Newkirk model. The PL model can be adjusted to any of these models by varying its parameters. The $n_{0}, h_{0}$ parameters are redundant; we have split them to clarify their physical meaning. The $x$ variable in the radial direction is $x \approx(r-1) R_{\odot}$. The PL model with $\delta=2, n_{0}=2.8 \times 10^{8} \mathrm{~cm}^{-3}$ agrees within $\pm 30 \%$ with the Newkirk model at $r=(1.2-9) R_{\odot}$, which is important in anaylses of type II bursts. The parameters of the PL model can be adjusted to the Saito model for various $\phi$ as well, which is important in analyses of CMEs. A single PL model with a direction-dependent $\delta$ provides a convenient alternative to complex involvement of various density models and allows one to account for individual properties of active regions as well as highly disturbed conditions just before the onset of a wave. 

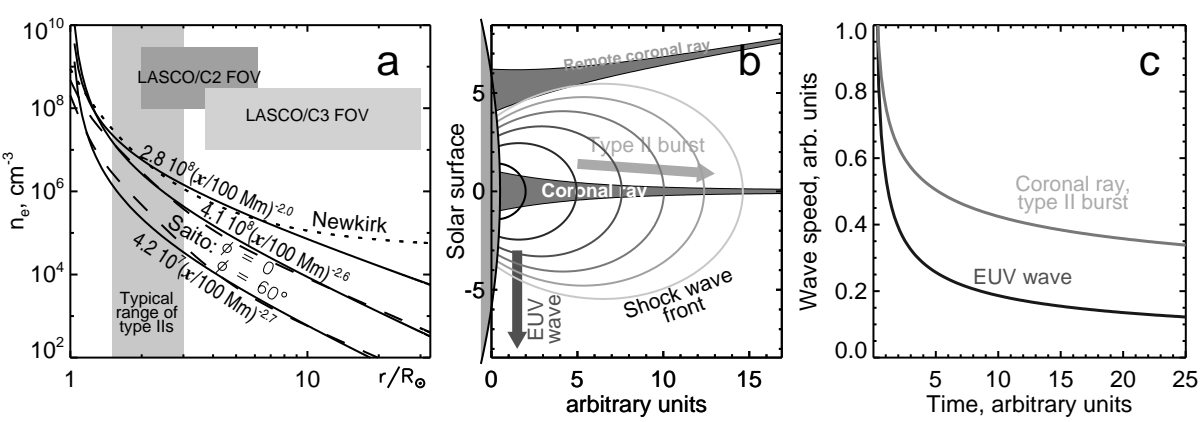

Figure 1. a) Coronal density models of Newkirk (dotted) and Saito (dashed, for latitudes $\phi=0^{\circ}$ and $\phi=60^{\circ}$ ). The solid lines represent the power-law model with different parameters to fit the Newkirk model at $r<9 R_{\odot}$ and the Saito model within the LASCO/C2 \& C3 fields of view shown with shading. The vertical shaded region indicates the typical range of type II bursts. b) A cartoon illustrating the relation between a type II burst and an EUV wave. A narrowband type II emission is generated by a shock front propagating in a distinct extended narrow structure like a coronal ray. A near-surface EUV wave runs slower. (c) Speed-time plots of the EUV wave and the type II emission site.

\subsection{Shock Waves and Type II Bursts}

Assuming $\delta$ to depend on a propagation direction, we get an approximation for a shock of an intermediate intensity propagating in an anisotropic medium (Figure 1b). A quasi-isotropic shock wave propagating in homogeneous corona can only cause drifting continuum radio emission. A strong narrowband harmonic type II burst can appear if the shock front passes along a lengthy structure like a coronal streamer (e.g., Reiner et al., 2003; Mancuso and Raymond, 2004). The cumulation effect increases the density jump in vicinities of the streamer's current sheet and intensifies radio emission (Uchida, 1974). The situation resembles a flare process running along a coronal ray (Uralova and Uralov, 1994). The difference between the horizontal and vertical directions explains a relation between the speeds of an EUV wave and a type II burst (Figure 1c). The upwards speed is higher than the surface one, because the wave center rises.

Even if a shock wave appears at a zero height, its front rapidly becomes convex and tilted towards the solar surface (Figure 1b). This front shape has been actually observed by Hudson et al. (2003) and is consistent with observations discussed by Warmuth et al. (2004b). If a convex shock front encounters a remote coronal ray, then the intersection site bifurcates, and its parts move along the ray in opposite directions ( $c f$. Mancuso and Abbo, 2004). The contact corresponds to an infinite drift rate followed by bidirectional drifts to resemble a direct bracket "(" in a dynamic spectrum. Note that dynamic spectra present a combination of emissions originating at different sites, so that the intensities are summed.

In Section 3 we reconcile kinematics of "EIT waves" and drift rates of corresponding type II bursts in terms of our approach based on the self-similar shock approximation (Section 2.1). We apply power-law curves with the same onset time to both spectral domains (hereafter 'shock-PL fit'). The density falloff index in a streamer determining the drift rate of a type II burst is expected to be $\delta \sim 2$. Real lateral density falloffs in a streamer should be steeper than along its 
axis. Otherwise, streamers would not be visible in homogeneous corona. Thus, real shock fronts should be oblate at medium distances. The density falloff for an EUV wave escaping from an active region can be $0<\delta<3$. For possible wave signatures in CMEs, the density falloff index is expected to be close to the Saito model (2), i.e., $\delta \sim 2.6$ at moderate latitudes and steeper at higher latitudes.

\subsection{Fit of Presumable Wave Signatures}

We fit the drift rate of a type II burst manually. The onset time $t_{0}$ of a wave can be approximately estimated from observations. We take parameters of the plasma density model $n_{0}=5.5 \times 10^{8} \mathrm{~cm}^{-3}, h_{0}=100 \mathrm{Mm}$, and $\delta$ according to the considerations in the preceding Section. One more input parameter is a reference frequency $f_{\text {obs }}$ of a band with a harmonic number $N_{f}$ (usually 1 or 2 ) actually observed in a dynamic spectrum at a time $t_{1}$. The corresponding plasma number density is $n_{1}=\left[f_{\text {obs }}\left(t_{1}\right) / N_{f} /\left(0.9 \times 10^{4}\right)\right]^{2}$, and the height is $x_{1}=h_{0}\left(n_{0} / n_{1}\right)^{1 / \delta}$. Then the height-time plot of the shock tracer is calculated as $x(t)=x_{1}\left[\left(t-t_{0}\right) /\left(t_{1}-t_{0}\right)\right]^{2 /(5-\delta)}$, the corresponding density variation as $n(t)=n_{0}\left[x(t) / h_{0}\right]^{-\delta}$, and the outline of both bands of the type II burst as $f(t)=[1,2] \times 0.9 \times 10^{4}[n(t)]^{1 / 2}$. By adjusting $\delta$ and $t_{0}$ in sequential attempts, we endeavor to approach a best outline of the bands. Uncertainties provided by the routine typically do not exceed $1 \mathrm{~min}$ for $t_{0}$ and 0.2 for $\delta$. The spectrum can be coordinated with measured heights by adjusting $n_{0}$, as usually done.

To fit presumable traces of shocks in coronagraph images (in this paper we use measurements from the CME Catalog), we employ two ways. The first way is a manual fit similar to the routine outlined in the preceding paragraph. Input parameters are starting estimates of $\delta$ and $t_{0}$, the heliocentric distances of the wave origin $r_{0}$ and the wave front $r_{1}$ measured at a time $t_{1}$. The initial approximation of the height-time plot is $r(t)=\left(r_{1}-r_{0}\right)\left[\left(t-t_{0}\right) /\left(t_{1}-t_{0}\right)\right]^{2 /(5-\delta)}+r_{0}$. Then sequential attempts are made to approach a best fit. The second way employs a $\log -\log$ height-time plot, which is a straight line for a power law. We use a second-order fit and iteratively vary $\delta$ and $t_{0}$ to minimize the second-order term. One should be aware of the fact that the major but unknown uncertainties can be due to identification of the wave front in coronagraph images.

\section{Observations}

\subsection{Event 1: 13 July 2004}

This event (Figure 2) was associated with an eruptive M6.7 flare (00:09-00:23, all times hereafter are UT) in active region 10646 (N13 W46) and a CME observed with SOHO/LASCO (Brueckner et al., 1995) after 00:54. Two parts of the CME (Figure 2b,c) are listed in the CME Catalog as two CMEs measured at position angles (PA) of $294^{\circ}$ and $213^{\circ}$. A type II burst was recorded in three observatories. The three estimates of the shock speed progressively decreased in time.

Grechnev et al. (2008) revealed signatures of a probable blast wave as an $\mathrm{H} \alpha$ Moreton wave (gray in Figure 2a) and an EUV wave (white). Both disturbances 


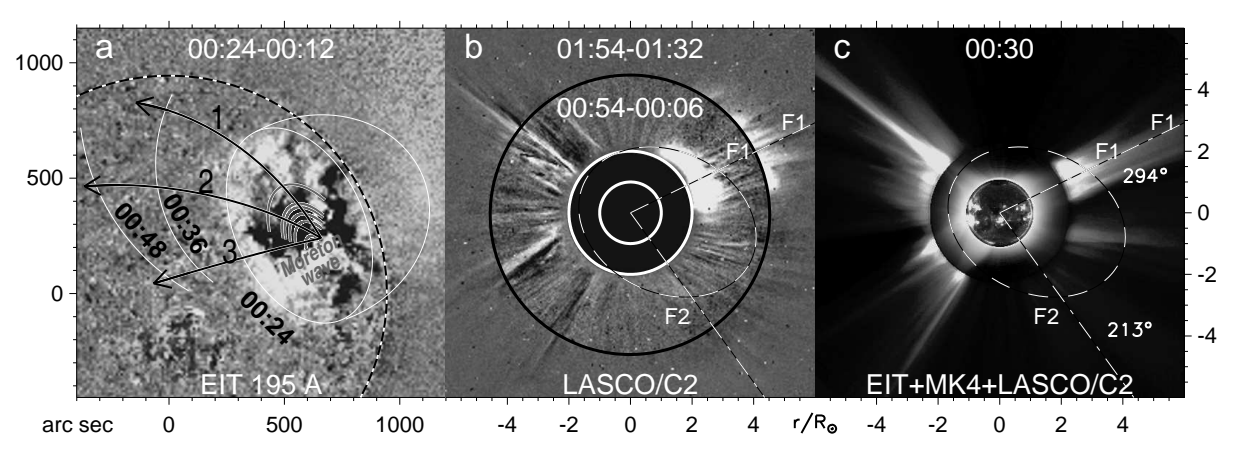

Figure 2. The 13 July 2004 event. a) The Moreton wave (gray) and EUV wave (white) fronts superposed on the EIT $195 \AA$ difference image. The black arcs 1, 2, and 3 trace the directions of measurements. The dotted circle denotes the limb. (b) The leading part of the CME observed in two LASCO/C2 difference images at 00:54 and 01:54 separated with the black circle. c) The non-disturbed corona in a combined image composed from non-subtracted EIT $195 \AA$, Mark4, and C2 images. The dashed ovals in (b) and (c) outline the CME in the 00:54 image. The dash-dotted straight lines in (b) and (c) mark the position angles at which the measurements listed in the CME Catalog were carried out for the fastest features F1 and F2. The axes show the distances from the solar disk center in arc seconds (a) and in solar radii (b and c).

were kinematically close to each other and to a kinematical curve expected for a lower trail of a decelerating coronal blast wave. Its exciter was not discussed. The authors proposed that the decreasing estimates of the shock speed reflected deceleration of a single shock wave, but they did not consider the type II burst.

Pohjolainen, Hori, and Sakurai (2008) [afterwards PHS] analyzed the type II burst in this event, but could not reconcile the overall drift with propagation of a single shock wave. The authors proposed that two shock waves were excited, one by a flare blast, and the second one by an expanding loop, a part of a CME.

We carried out an additional analysis of this event, measured kinematics of an eruptive system in order to find out a probable origin of a shock wave(s), and to reconcile its (their) propagation with the EUV/Moreton waves and the CME.

\subsubsection{Eruptive System}

Figure 3 shows the eruptive system: a leading bright feature 'bf', two filament segments ' 1 ' and '2', and several eruptive loops, of which one ('loop') was conspicuous and thus will be discussed henceforth. Long exposure times (33-46 s) caused a blurring of fast features, e.g., a jetlike appearance of the bright feature.

To measure expansion of a feature, we outline it with an oval arc that allows us to trace it, even if its leading edge is difficult to detect. The obtained distancetime plot is used as a starting estimate. Then we choose a regular function to match the distance-time plot and estimate its parameters. Using the analytic fit, we calculate expected distance-time points, compare them with observations, and improve the fit. All kinematical plots are calculated by means of integration or differentiation of the analytic fit rather than measurements. Our ultimate criterion is to reproduce the motion of an analyzed feature. Observational limitations do not allow us to reveal a detailed time profile of acceleration, and we describe it instead with a smooth bell-like function. We use a Gaussian time 


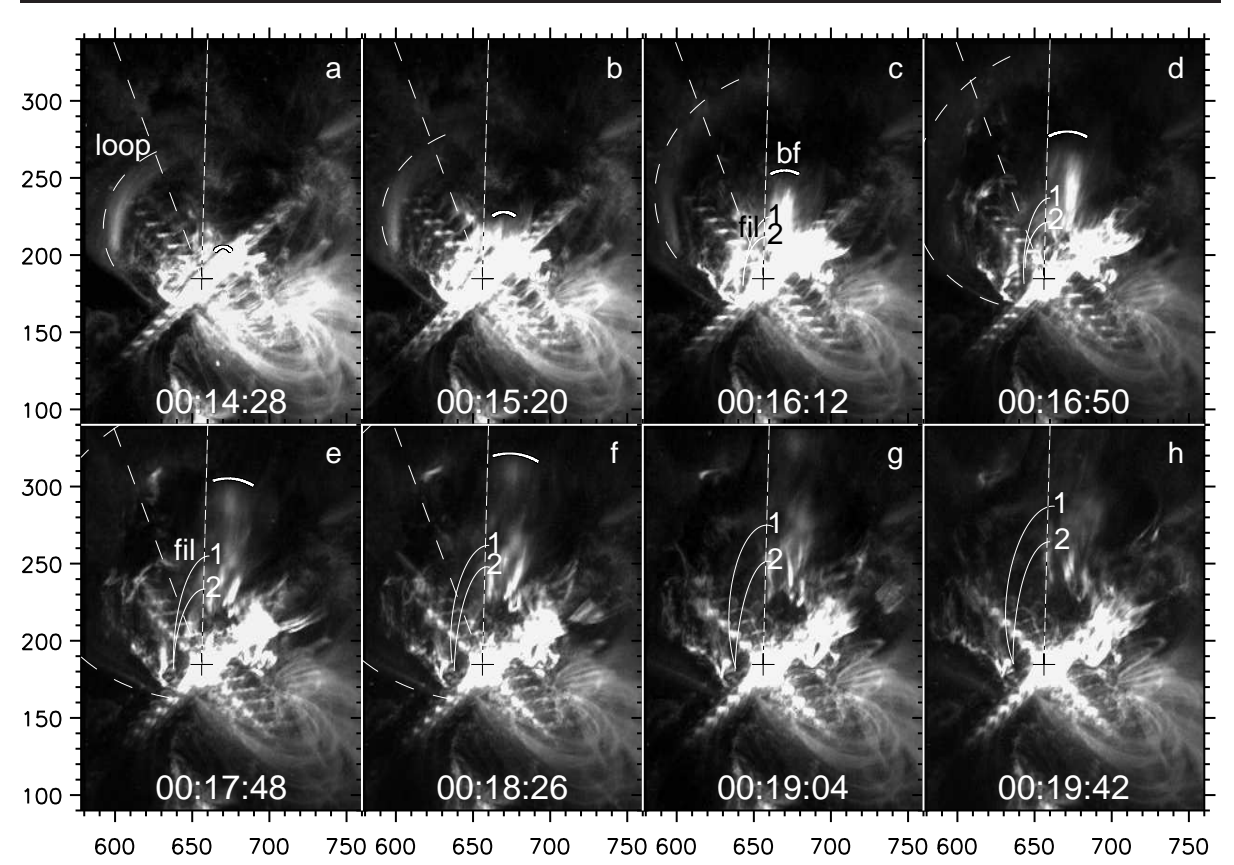

Figure 3. The eruptive system in the 13 July 2004 event (TRACE, $173 \AA$ ). The cross marks the initial position of the filament. Oval arcs outline the eruptive loop (dashed), filaments (solid), and bright feature (short arc). The broken lines denote the expansion directions of the loop (dashed) and the filament (dotted). Axes show arc seconds from the solar disk center.

profile (see Wang, Zhang, and Shen, 2009). Then acceleration $a$ is

$$
a=\left(v_{1}-v_{0}\right) \exp \left\{-\left[\left(t-t_{0}\right) / \tau_{\text {acc }}\right]^{2} / 2\right\} /\left(\sqrt{2 \pi} \tau_{\text {acc }}\right)
$$

Here $\tau_{\text {acc }} \sqrt{8 \ln 2}$ is a full width at half-maximum of the acceleration time profile, which is centered at the $t_{0}$ time; $v_{0}$ and $v_{1}$ are velocities at the onset and end of the acceleration stage. In cases of a more complex kinematics, we use a combination of Gaussians and adjust their parameters manually.

Uncertainties are mainly determined by difficulties to identify and trace a feature in question. For this purpose we use both non-subtracted and difference images processed in various ways and improve results in sequential attempts. The worst traceable feature in this event is the eruptive filament, which appears as a semitransparent dark feature, whose segments 1 and 2 are faintly visible in Figure 3. We therefore present the measurements of the filament in more detail.

The results of plane-of-sky measurements are shown in Figure 4 for the loop and bright feature (left) and for the filament segments (right). The dashed curve in Figure 4d was calculated from the dashed acceleration profile in Figure $4 \mathrm{f}$ to match an upper envelope of the measured positions of segment 1 . The dashed curve in Figure 4e is a corresponding velocity profile. The dotted curves in Figure $4 \mathrm{~d}-\mathrm{f}$ correspond to a lower envelope of the positions of segment 2 . The black solid curves correspond to an average height - time plot. The horizontal bars show the exposure durations. We consider the maximum acceleration of the middle 

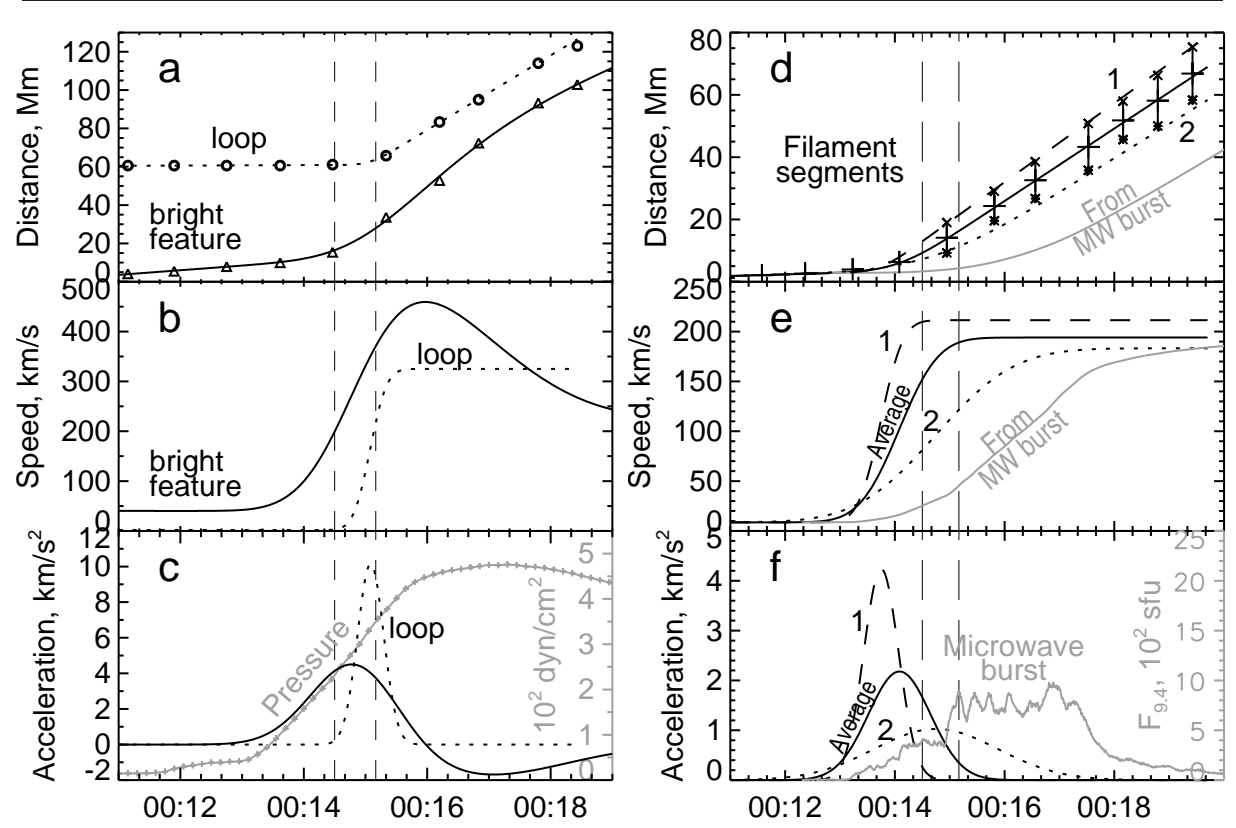

Figure 4. Kinematical plots of the eruptions in the 13 July 2004 event: the loop and bright feature (left) and the filament segments 1 and 2 (right; the solid curves correspond to averages between 1 and 2). Symbols mark the measured plane-of-sky distances, and curves represent their fit. The gray curve in panel (c) displays the plasma pressure computed from GOES SXR fluxes. The gray curves in panels $(\mathrm{d}-\mathrm{f})$ show kinematical plots calculated by assuming the correspondence of the acceleration plot to the microwave one. The vertical dashed lines delimit the start time of the wave estimated by Grechnev et al. (2008).

part of the filament to reach about $2 \mathrm{~km} \mathrm{~s}^{-2}(-60 \%,+120 \%)$, and its probable speed and positions to be close to the solid black curves, being within the dotted and dashed boundaries. The bright feature and eruptive loop are visible much better, their leading edges are well defined, and therefore uncertainties of their positions are significantly less. The estimated height-time profiles match the measurements. The main uncertainty here is due to temporal undersampling, which is only crucial for the estimate of acceleration of the loop.

When the measurements began, the bright feature already rose that probably corresponded to the initiation phase, which started at about 00:07 according to soft X-ray (SXR) GOES data. A strong acceleration started at about 00:13, reached $\approx 4 \mathrm{~km} \mathrm{~s}^{-2}$, and then changed to deceleration. The loop was static by 00:14:30; after 00:15:10 its speed sharply changed to $V_{\text {loop max }} \approx 320 \mathrm{~km} \mathrm{~s}^{-1}$ and did not increase afterwards. The last measured point hints at a possible later deceleration. The transition from the initial zero speed to a final one occurred between two samples. Hence, the maximum acceleration of the loop could well exceed $10 \mathrm{~km} \mathrm{~s}^{-2}$. All components of the corresponding CME decelerated (Grechnev et al., 2008), so that the eruption resembled an explosion with a strong impulsive acceleration followed by continuous decreasing deceleration.

Figure 4c also shows the plasma pressure computed from SXR GOES fluxes and a source size of $15 \mathrm{Mm}$ found from RHESSI (Lin et al., 2002) images. The 
pressure gradually rose while the bright feature suddenly started to decelerate. Thus, the flare pressure was unlikely a driver of either the eruption or the wave, whose estimated start time is delimited with vertical dashed lines.

The filament started to rise nearly simultaneously with the bright feature. However, both the acceleration and speed of the bright feature were higher, and it surpassed the filament (see Figure 3). The nature of this feature is difficult to identify. In some images it resembles an arcade surrounding the filament, but initially it seems to be located below the filament. This feature might be also one more filament, which brightened due to heating. An additional possibility is suggested by a scenario proposed by Meshalkina et al. (2009): this feature might be a small-scale magnetic rope whose eruption destabilized the filament.

The gray curves in Figure $4 \mathrm{~d}-\mathrm{f}$ show the kinematical plots calculated under the assumption that the acceleration plot corresponded to the $9.4 \mathrm{GHz}$ light curve (Nobeyama Radio Polarimeters, Torii et al., 1979; Nakajima et al., 1985; ftp://solar.nro.nao.ac.jp/pub/norp/xdr/). Microwave bursts are known to be close in shape to hard X-ray (HXR) ones, while accelerations of eruptions have been found to be close to HXR bursts (e.g., Temmer et al., 2008). The plots calculated from the microwave burst lag behind the actual plots of the filament by about two minutes indicating that, most likely, the flare was caused by the eruption. This fact suggests that the eruptive filament accelerated almost independently of the flare reconnection rate and HXR emission, at least, in this event.

There are two options regarding a relation between the bright feature and the loop. One possibility is that $\approx 1.5-2$ minutes after the start of the acceleration of the bright feature, the loop suddenly and independently underwent much higher impulsive acceleration. Alternatively, the loop was expelled by a shock front that appears to be more probable. For the latter case, the strength of the shock can be estimated. The Mach number is $M=V_{\text {sh }} / V_{\text {fast }}$, where $V_{\text {sh }}$ is a shock speed, and $V_{\text {fast }}$ is a fast-mode speed. The shock speed $V_{\text {sh }}$ at the onset of the loop motion can be roughly estimated from a PL fit (Grechnev et al., 2008) to be about $1000 \mathrm{~km} \mathrm{~s}^{-1}$, but it is rather uncertain because of insufficient temporal coverage by TRACE images and their long exposure times. The fast-mode speed can be estimated from an expression $V_{\mathrm{sh}} \approx V_{\text {fast }}+\kappa U_{\mathrm{sh}} / 2$, where $\kappa$ is a coefficient determining the steepening rate of the wave front. This coefficient, $1 / 2 \leq \kappa \leq$ $3 / 2$, depends on plasma beta and the propagation direction. We take the speed of the loop as the gas speed behind the shock front, $U_{\mathrm{sh}}=V_{\text {loop max }} \approx 320 \mathrm{~km} \mathrm{~s}^{-1}$ (see Figure $4 \mathrm{~b}$ ). With these quantities and $\kappa \approx 3 / 2$ for the wave propagation perpendicular to the magnetic field in low-beta plasma, the Mach number is $M \approx 1.3$. One might suppose that the steepening time was about the interval between the peaks of the solid and dotted curves in Figure 4c corresponding to the acceleration profiles of the bright feature and the loop, i.e., about $0.5 \mathrm{~min}$.

We extrapolated coronal magnetic fields from a SOHO/MDI magnetogram using a package of Rudenko and Grechnev (1999) based on a potential approximation (Rudenko, 2001). The result showed that the eruptive loops were strongly tilted to the photosphere, and their tops were at a height of about $30 \mathrm{Mm}$. With a height of the pre-eruptive filament of $>10 \mathrm{Mm}$, the shock front hit the loop almost horizontally, and the estimated Mach number is related to the horizontal direction, while in the vertical direction the shock was probably stronger. 


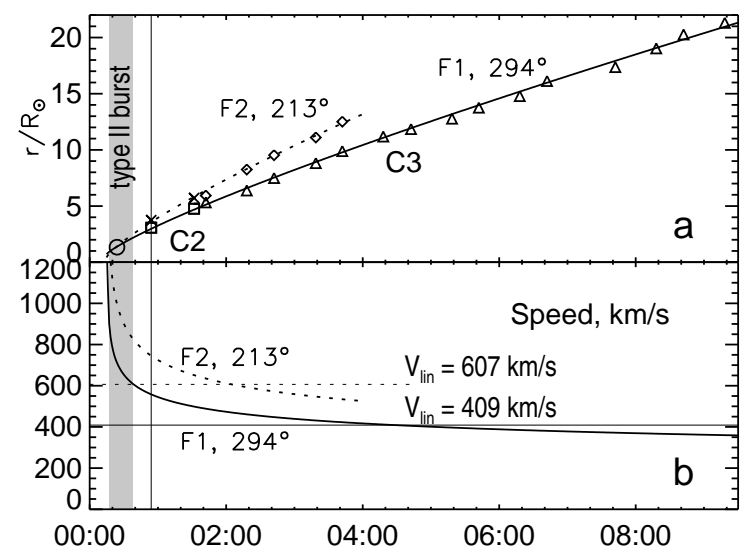

Figure 5. a) Measurements from the CME Catalog for the F1 and F2 CME components fitted with the shock-PL model, $\delta \approx 2.6$. The circle denotes the position of the off-limb EUV wave at $294^{\circ}$. b) Speed-time plots corresponding to each fit. Linear-fit speeds are also specified. Shading denotes the interval in which the type II burst was observed.

Thus, the loop itself was most likely passive, and its motion was driven by the shock wave. All observed products of the eruption monotonically decelerated starting from TRACE observations and up to LASCO/C3 ones. The loop therefore is unlikely to have excited the second shock wave, as PHS hypothesized.

\subsubsection{Moreton/EUV Wave, Type II burst, and CME}

Grechnev et al. (2008) could not find out from kinematics if the leading edge of a coronal transient observed by LASCO (see Figure $2 \mathrm{~b}$ ) was a mass ejection or a trace of a wave. Comparison with a non-subtracted image of the corona before the CME in Figure 2c suggests its spiky leading fringe to be coronal rays deflected by a wave (pronounced for feature $\mathrm{F} 1$ at $\mathrm{PA}=294^{\circ}$ ). Vourlidas et al. $(2003)$ and Magdalenić et al. (2008) interpreted such phenomena as signatures of shocks. Assuming that the CME leading edge was due to the wave, we outlined the measurements from the CME Catalog with a shock-PL fit. The calculated curves with $\delta \approx 2.6$ corresponding to the Saito model agree with the measurements (Figure 5). The decreasing speeds computed from these height-time plots at distances corresponding to the $\mathrm{LASCO} / \mathrm{C} 2$ and $\mathrm{C} 3$ fields of view agree with the linear-fit speeds estimated in the CME Catalog, while within the interval when the type II burst was observed the speeds are higher by a factor of $2-2.5$.

Figure 6c shows a type II burst recorded by the HiRAS radio spectrograph and our shock-PL fit of both the fundamental and second-harmonic emission (solid lines). We use the density falloff index $\delta=2.1$, which is close to the Newkirk model expected for a streamer. Features QF1 and QF2 are open to question; their presence hinted PHS at two different shock waves. The dotted lines approximately reproduce the outline of PHS following the logic suggested by their Figs. 4 and 6 . They correspond to fixed velocities of the type II exciters. However, a flare blast wave proposed by the authors is expected to decelerate: just our outline corresponds to a freely propagating blast wave. 


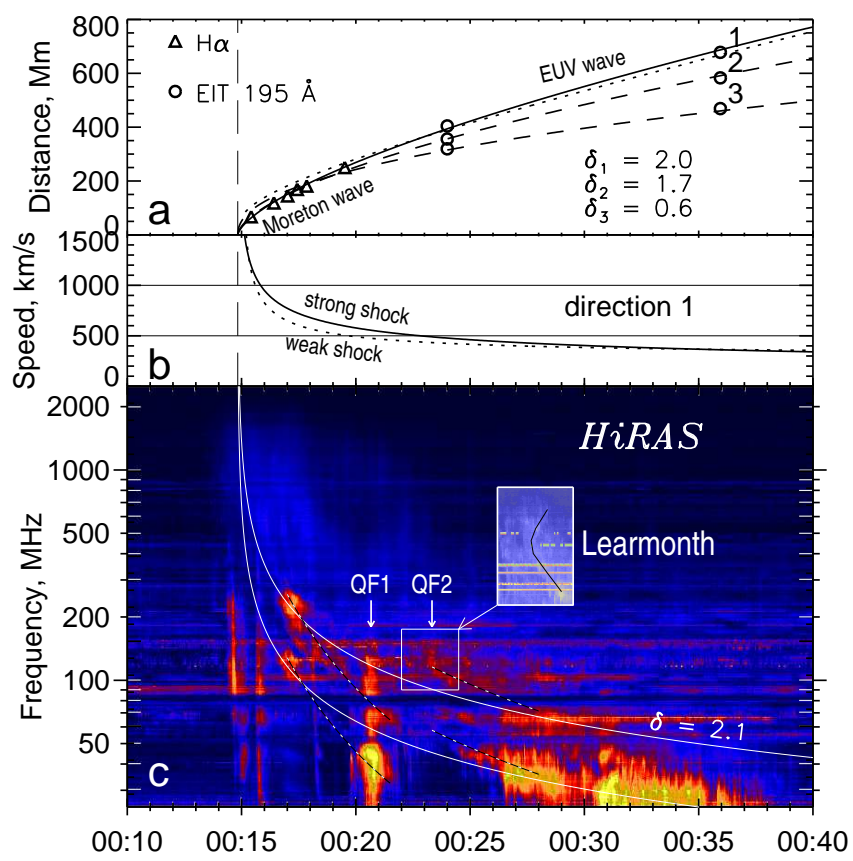

Figure 6. The 13 July 2004 event. Height-time (a) and velocity-time (b) plots of the Moreton and EUV waves, and (c) the fit of the type II burst $(\delta=2.1)$. Qf1 and QF2 are features open to question. A reverse drift of Qf2 is detectable in the inset which shows a portion of the dynamic spectrum recorded in Learmonth with a higher spectral resolution.

The solid shock-PL fit outlines the whole slowly drifting structure from the decimetric range up to the lowest frequency. A question remains about features QF1 and QF2. The former feature with an uncertain harmonic structure does not seem to favor the dotted outline relative to the solid one. The weaker QF2 feature, which PHS considered as the onset of the second type II burst, indeed seems to have a harmonic structure. Its shape in the higher-resolution spectrum recorded at the Learmonth station (US Air Force RSTN) resembling "(" outlined with a black arc suggests an encounter of a shock wave with a dense structure (see Section 2.2). These facts support association of the type II burst with a single decelerating shock wave. The drifting continuum, which PHS found to start at 00:13 (confirmed by the acceleration profile in Figure 4f), might be due to emission from outside of a pre-shock region expanding towards a decreasing density or, alternatively, from inside of the expanding region with a progressively depleting density, as PHS proposed. Compression of the environment in the preshock interval from 00:13 to about 00:14:50 might have produced an excessive plasma density, which we describe with a radial power-law falloff.

For comparison we show in Figure 6a the distance-time measurements of the Moreton wave (triangles) and the EUV wave (open circles) from Grechnev et al. (2008) along with shock-PL plots for the three directions 1, 2, and 3 denoted in Figure 2a, and the dotted plot of a spherical weak shock propagating in uniform plasma calculated by using expressions of Uralova and Uralov (1994). Figure $6 \mathrm{~b}$ presents the speeds for the strong (solid) and weak (dotted) shock 
approximations along direction 1. Both approximations are close to each other far from the eruption center $\left(r \gtrsim R_{1} \approx 200 \mathrm{Mm}\right)$ being somewhat different at shorter distances. The shock wave propagating along the surface probably became weak at $r \gtrsim R_{1}$, when it left the active region and entered quiet Sun's areas where the coronal density and the fast-mode speed were nearly constant, i.e., $\delta \rightarrow 0$. Closer to the eruption site $\left(r<R_{1}\right)$, the plasma density presumably had a power-law falloff, and the shock was not weak. These conditions seem to favor the self-similar shock approximation, in which $i$ ) the shock propagation speed is proportional to its intensity and does not depend on the fast-mode speed, and $i i$ ) the wavelength is equal to the distance passed by the wave, i.e., its duration increases. The density falloff of $\delta<3$ within an active region and at its periphery corresponds to deceleration and damping of such a shock. The limit of a strong shock is a convenient idealization to describe the formation stage of a single shock wave, which propagates far from its source. A real forming shock wave does not seem to be so strong that the decrease of the fast-mode speed could be neglected when the shock leaves the active region. This issue is beyond our scope. We only note that formation of the shock discontinuity in a disturbance produced by an impulsive piston presumably completes (and its intensity reaches maximum) in a region where the falloff of the fast-mode speed is steepest.

Note that deceleration of the EUV wave sweeping over the quiet solar area was stronger towards the equator, as comparison of the three fronts in Figure 2a for the $1-3$ directions shows. This is expected for a strong shock, whose deceleration is determined by the density distribution, which is maximum at the equator (see the Saito model). This is also expected for a weak shock, whose propagation is governed by the Alfvén velocity decreasing towards the equator due to both the density distribution and the dipole magnetic field of the Sun.

Our analysis of Event 1 has revealed a probable excitation of a single wave by an impulsively accelerated eruption and steepening into a shock within one minute. Then the wave freely propagated like a decelerating blast wave and probably formed the leading edge of the CME. Our results are consistent with the conclusions of PHS about the role of a rapidly expanding eruption, formation of the shock wave at a very low altitude, and their estimates of the shock speed.

\subsection{Event 2: 1 June 2002}

Meshalkina et al. (2009) revealed a possible coronal shock wave presumably excited by a collision of an eruptive magnetic rope with a magnetic obstacle in the 1 June 2002 event. An M1.5 flare (S19 E29) started at 03:50 and lasted only $11 \mathrm{~min}$. SOHO/EIT carried out the 'High cadence 195' program, and LASCO did not observe at that time. Figure 7 shows an off-limb EUV wave in this event.

Figure $8 \mathrm{a}-\mathrm{f}$ presents the eruption with extreme outlines (dotted and dashed) of its leading edge found from two sets of images processed in different ways. Figure $8 \mathrm{~g}-\mathrm{i}$ presents plane-of-sky measurements of kinematics using the same technique as for Event 1. The eruption accelerated up to $\approx 7 \mathrm{~km} \mathrm{~s}^{-2}$ and then decelerated. The deceleration might be overestimated, because the eruption started to disintegrate and become transparent. Similarly to Event 1, acceleration occurred during the rise of the HXR burst recorded with RHESSI, while the plasma pressure gradually increased all the time. 


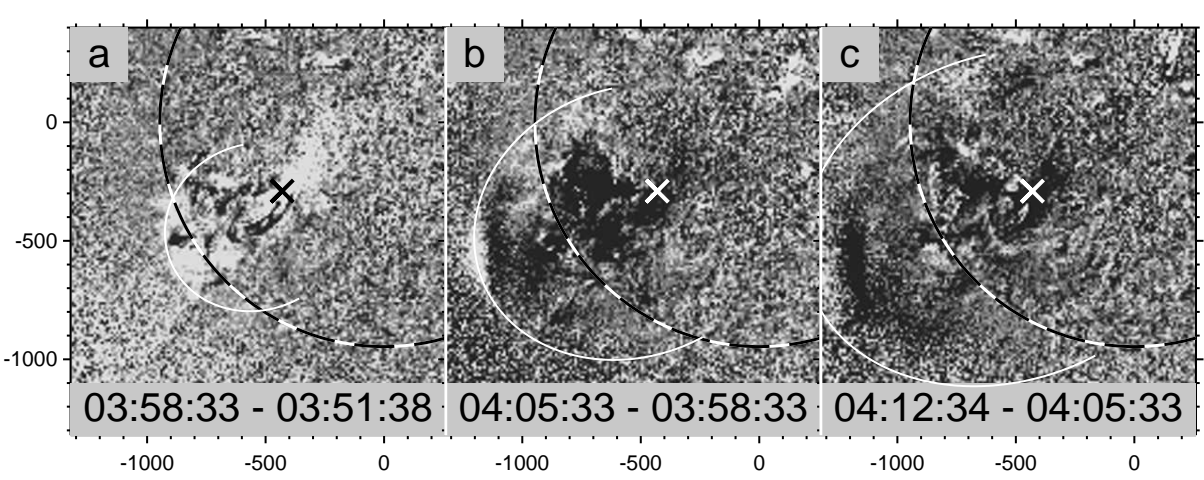

Figure 7. An EUV wave (outlined with ovals) on 1 June 2002 in EIT 195 Å running-difference images. The dashed circles denote the solar limb. The slanted cross marks the flare site.
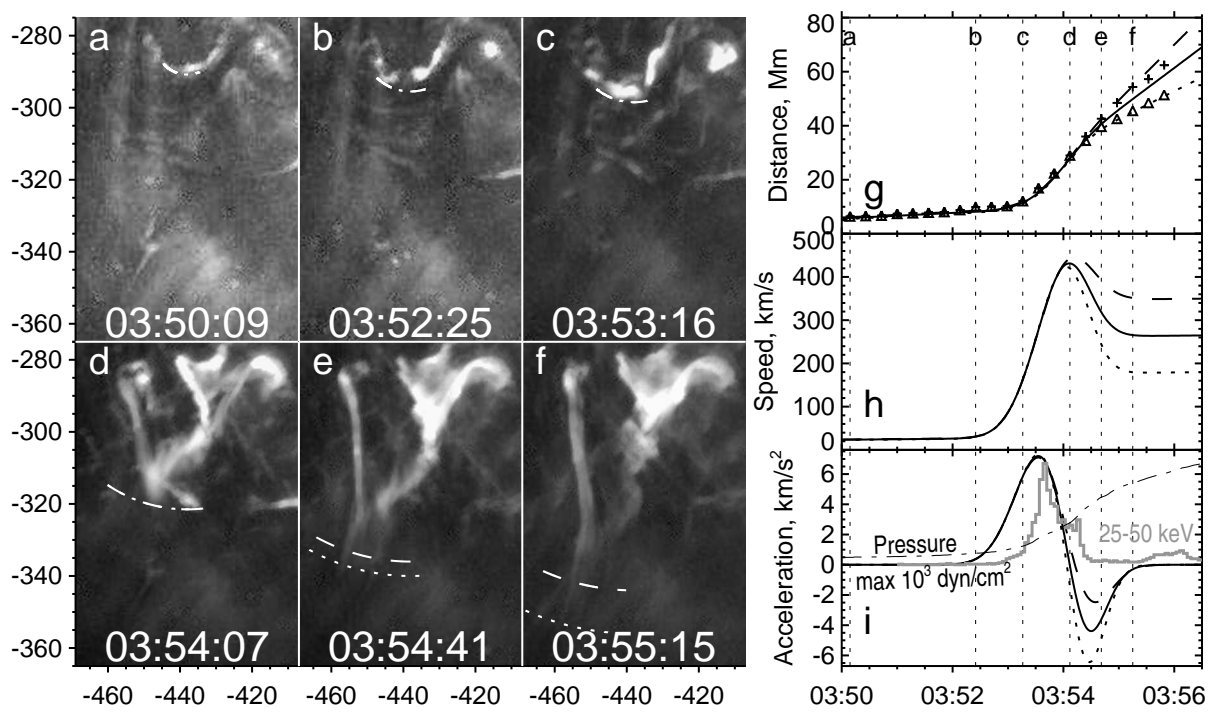

Figure 8. The eruption in TRACE $195 \AA$ images $(\mathrm{a}-\mathrm{f})$ and its kinematics $(\mathrm{g}-\mathrm{i})$. The dotted and dashed lines correspond to extreme outlines of the eruption. Panel (i) also shows a $25-50 \mathrm{keV}$ RHESSI time profile (gray) and the plasma pressure calculated from GOES data (dash-dotted). The vertical dotted lines mark the observation times of images $\mathrm{a}-\mathrm{f}$.

Figure 9 shows kinematics of the EUV wave $(a, b)$ and the type II burst (c) similarly to Figure 6 . To reveal the harmonic structure of the burst, we use again the record made in Learmonth (the inset). The burst consisted of two pairs of emission bands with frequency ratios in pairs of 2.0 and $\approx 1.5$ between the pairs. The two pairs of bands resemble band-splitting usually interpreted as the plasma radiation from the regions upstream and downstream of a shock. However, the relative split is atypically large for the metric range (Vršnak et al., 2001). Alternatively, this situation suggests propagation of the shock front along two streamers located close to each other. We outline the burst structure with two harmonic pairs of power-laws 1 and 2 with ' $f$ ' indicating the fundamental emission and ' $\mathrm{h}$ ' the second harmonic. The difference in $\delta$ (2.4 and 2.6) might be 


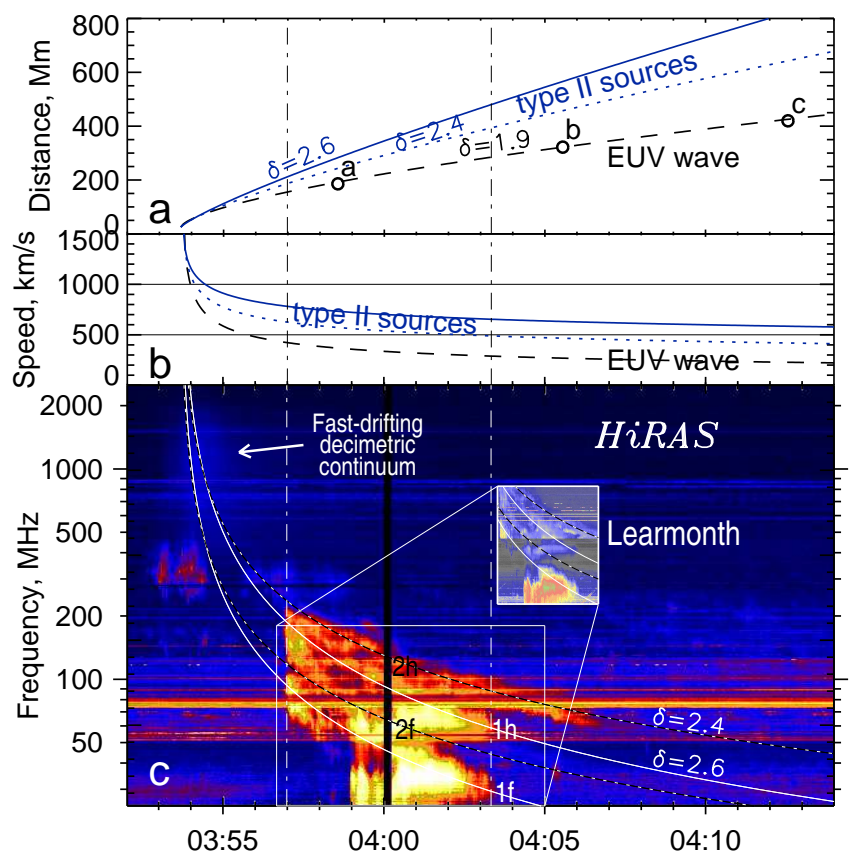

Figure 9. The 1 June 2002 event. Height-time (a) and velocity-time (b) plane-of-sky plots of the EUV wave (black) and the fit of the type II burst source (blue). Open circles in panel (a) represent the distances of the foremost wave fronts from the eruption center, and labels ' $a$ ' - 'c' correspond to the images in Figure 7. Panel (c) shows the dynamic spectrum with a shock-PL fit. The vertical broken lines delimit the interval of the type II burst.

due to differences of density falloffs in the streamers as well as different angles between the shock front and the axes of the streamers. The estimated wave onset time is 03:53:40, close to the acceleration peak time (Figure 8i).

Figure 9a shows height-time plots corresponding to the drift of the type II burst along with the EUV wave expansion. The type II emission was observed when its source was presumably located at heights from $190 \mathrm{Mm}$ (heliocentric distance of $\left.1.27 R_{\odot}\right)$ to $500-600 \mathrm{Mm}\left[(1.7-1.9) R_{\odot}\right]$, i.e., lower than usually assumed. Moreover, the outline of the shock front matches the fast-drifting decimetric continuum suggesting its relation to the shock wave, which presumably appeared still lower. The type II burst probably started when the shock front reached the streamer and ceased due to deceleration and damping of the wave.

\subsection{Event 3: 19 May 2007}

This event associated with a B9.5 flare at 12:48-13:19 (N07 W06) and a fast CME has been well studied due to efforts of several researches mostly from observations made with EUV Imager (EUVI) of SECCHI complex (Howard et al., 2008) on STEREO (Kaiser et al., 2008). Nevertheless, some questions remain.

Long et al. (2008) measured kinematics of the EUV wave, found distinct deceleration, and stated that the low velocities of "EIT waves" could be due to their temporal undersampling. The observations were found to be consistent with 


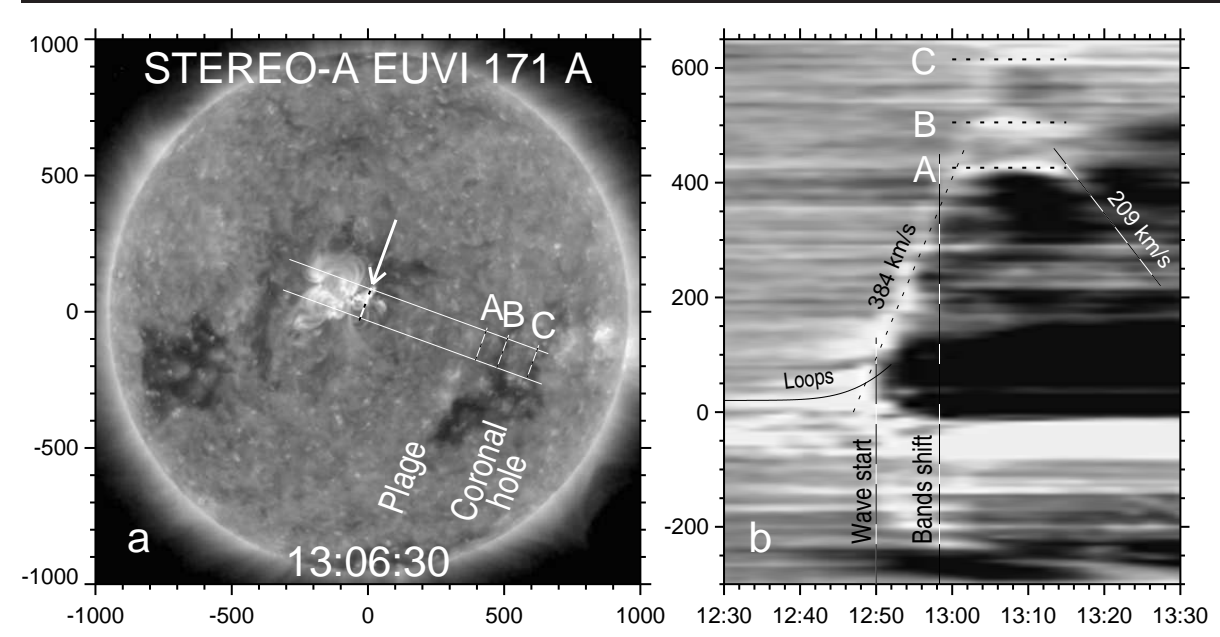

Figure 10. EUV wave observed with STEREO-A/EUVI at $171 \AA$ on 19 May 2007. A set of spatial profiles (b) computed from ratio images within the strip contoured in panel (a). The arrow points at the origin of measurements. The A, B, and C broken bars in both panels mark the reflection positions. The slanted broken lines in panel (b) outline the steepest slopes.

an impulsively generated fast-mode magnetosonic wave or propagating MHD shock. However, they revealed an initial acceleration of the disturbance from a nearly zero speed. This mismatches an expected behavior of an MHD wave.

Veronig, Temmer, and Vršnak (2008) also found deceleration of this disturbance indicative of a freely propagating MHD wave and revealed a wave reflection at a coronal hole. They assumed that the wave was initiated by the CME, because the associated flare was very weak and occurred too late to account for the wave initiation. They also revealed two eruptions following each other.

Gopalswamy et al. (2009) measured propagation of reflected wave fronts and considered the reflections as an argument in favor of a wave nature of EUV transients (see also Schmidt and Ofman, 2010). However, Attrill (2010) proposed that the reflections resulted from a misinterpretation of the running difference data and suggested instead that two EUV wave fronts developed during the event. Indeed, running differences reliably show only the outer boundaries of expanding disturbances, while the inner picture reflects all changes occurring between two images subjected to subtraction (Chertok and Grechnev, 2005).

To see whether or not the reflections actually occurred, we use a movie composed of non-subtracted $171 \AA$ images of STEREO-A/EUVI (euvi_ahead_171.gif in the electronic version of the paper). A backward motion suggesting a reflection is visible after 13:11 northeast from a plage region denoted in Figure 10a. We repeated the measurements of Gopalswamy et al. (2009), but without any subtraction. We only consider the first probable reflection in the direction exactly backwards, because it is difficult to reveal wave fronts in non-subtracted images of this complex event. Figure 10 corresponds to Figs. 1 and 2 from their paper. To enhance the sensitivity, we use in panel (b) spatial profiles computed as the sums over the width of each slice extracted from an image as panel (a) shows. Each image was normalized to a pre-event image at 12:16:30 (fixed-base ratios). 


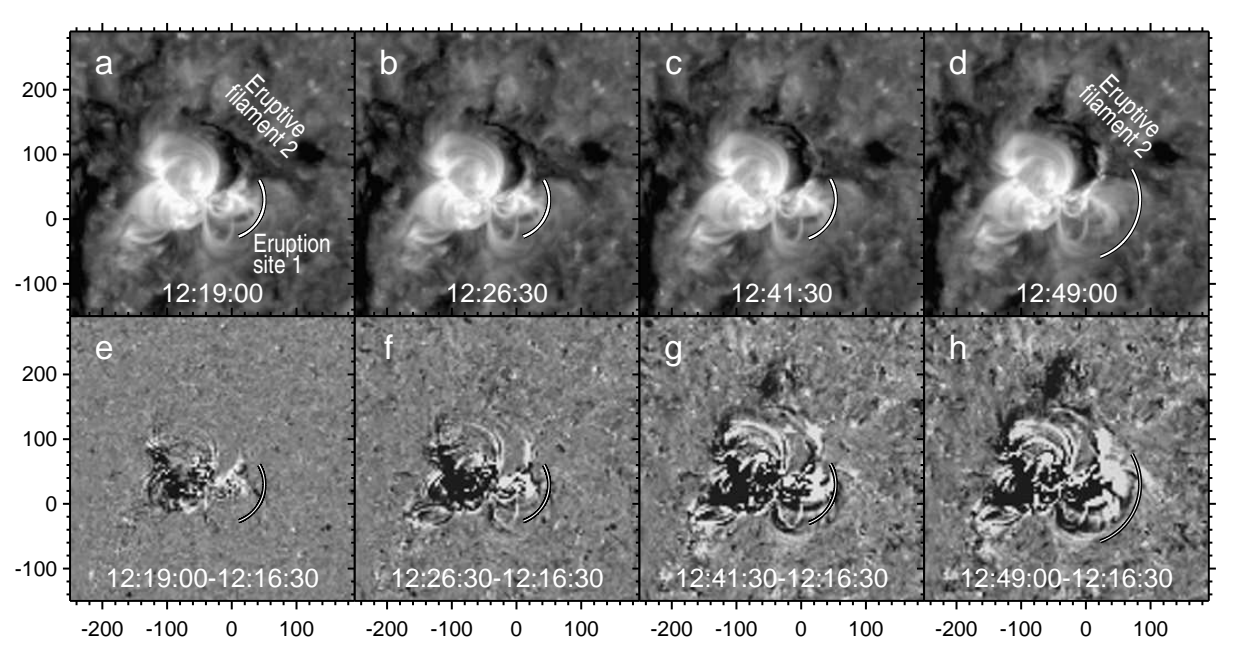

Figure 11. The first eruption on 19 May 2007 observed with STEREO-A/EUVI at $171 \AA$. Top: non-subtracted images, bottom: fixed-base differences. The arcs outline the foremost edge of the eruptive loop system according to the fit shown with the solid line in Figure 10b.

Figure 10b shows that the plage region inflected after arrival of the wave front and then returned back. A backwards motion from plage A is faintly visible after 13:14. Region B exhibited a weaker bend. It is not clear from which region of $\mathrm{A}$ and $\mathrm{B}$ was the wave reflected. The latter seems to be preferable, because continuations of both the direct and reflected slanted traces intersect farther from region $\mathrm{A}$, while a prolonged standing of the wave at region $\mathrm{A}$ is doubtful. The slanted broken lines represent the speeds found by Gopalswamy et al. (2009) and agree with the slopes in Figure 10b. Thus, the results of the authors were correct, at least, for the first reflection. The wave reflected backwards was considerably slower than the incident wave. This fact supports the shock-wave nature of the disturbance. Indeed, if an incident shock wave propagating with a velocity $V_{\text {inc }}$ sh encounters a semitransparent 'wall' like a coronal hole, then the shock reflected backwards is slower: $V_{\text {back sh }} \approx V_{\text {inc sh }}-V_{\text {gas }}$, where $V_{\text {gas }}$ is a velocity of the gas trailing the incident shock front. $V_{\text {gas }}$ can be up to the sound speed.

What does the accelerating part prior to 12:50 display? Figure 11 shows EUVI $171 \AA$ images with subtraction and without it. The outermost boundary of the expanding bright feature coincides with the edge of coronal loops visible in the earlier non-subtracted images. Then eruptive loops rapidly loose brightness due to expansion and become invisible in non-subtracted images. We conclude that the accelerating part measured by Long et al. (2008) was related to the expanding loops, while the decelerating part was related to the wave. It is difficult to distinguish an appearing wave, which brightens, from a piston (loops), which becomes transparent. For this reason the acceleration of the loops is uncertain within $120-270 \mathrm{~m} \mathrm{~s}^{-2}$; the outline in Figures 10 and 11 corresponds to $260 \mathrm{~m} \mathrm{~s}^{-2}$.

Figure 12a shows the measurements of the wave presented by Veronig, Temmer, and Vršnak (2008) and their shock-PL fit (thick blue curve). The wave start (12:50) corresponds to the early rise phase of the HXR burst (red, also from their paper). The power-law fit corresponds to the measurements of the authors 


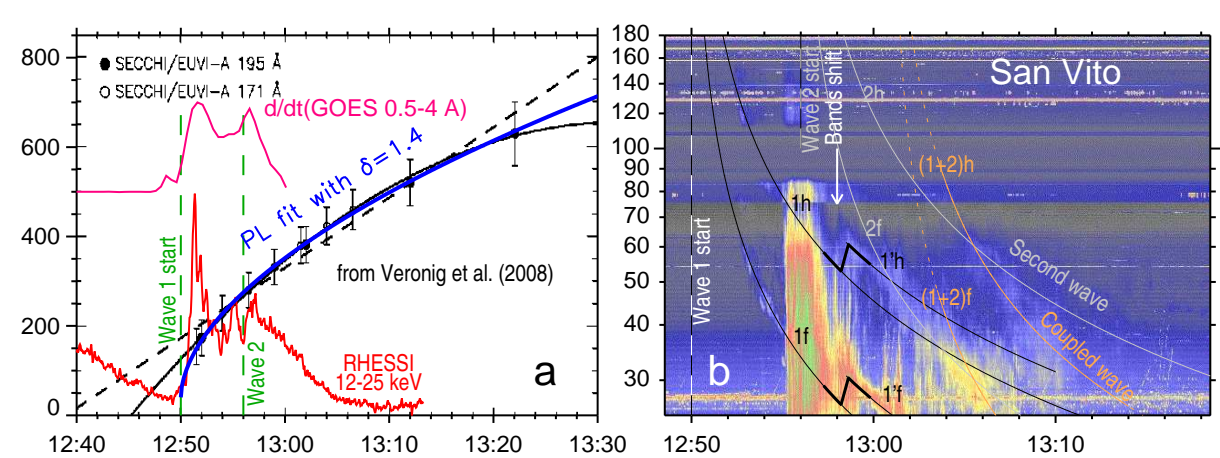

Figure 12. The 19 May 2007 event. a) Propagation of the EUV wave measured by Veronig, Temmer, and Vršnak (2008, black) along with an HXR time profile (red), a derivative of the SXR flux (pink), and a shock-PL fit (blue). b) Type II burst with shock-PL-fitted four pairs of bands. Paired bands are shown with the same colors. See details in the text.
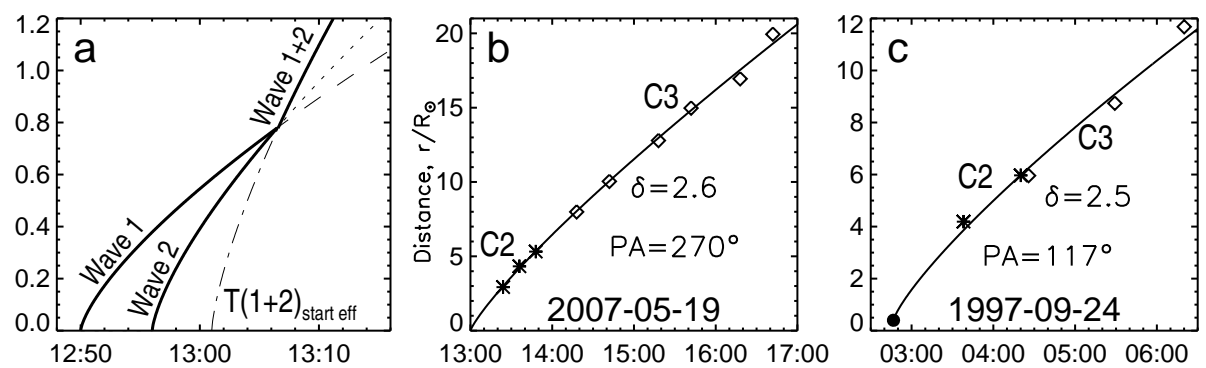

Figure 13. Coalescence of two shock waves (a) and height-time plots of CMEs observed on 19 May 2007 (b) and 24 September 1997 (c). Symbols present data from the CME Catalog, lines show their shock-PL fit. The filled circle in panel (c) denotes the origin of the wave.

better than their quadratic fit and the linear one. The eruption in this event also accelerated before the appearance of manifestations of flare reconnection.

The suggestion of Attrill (2010) about the second wave appears to be correct. The second eruption was probably triggered by the first one. Filament 2 activated at about 12:47 and erupted at 12:55-12:57 according to TRACE $173 \AA$ images. The HXR time profile was complex, but two distinct episodes are detectable in the derivative of the SXR flux recorded with GOES (pink in Figure 12a). The onset times of the two waves were about 12:50 and 12:56.

A shock wave trailing a preceding one must reach the leading front due to properties of shock waves; the two shock waves coalesce to produce a single shock front (and a weak backwards disturbance, which we are not interested in). Its speed is less than the sum of the initial fronts' speeds; however, the resulting shock is stronger and faster than either of the initial ones. So the slope of its distance-time plot is steeper than the initial waves had, and its virtual onset time is later than for either of the initial waves, as Figure 13a outlines.

Let us try to understand a complex dynamic spectrum in Figure 12b recorded in San Vito (USAF RSTN). A harmonic pair of weak bands $1 \mathrm{f}, 1 \mathrm{~h}$ is sometimes detectable after 12:52. A strong type III burst at 12:55-12:58 probably associated with the second eruption partially blocked the weaker bands $1 \mathrm{f}, 1 \mathrm{~h}$. Two stronger type II bands 1'f, 1'h appeared at 12:58. Their appearance looks like an 
inverse-N-like shift of the initial bands to higher frequencies (thicker outline in Figure $12 \mathrm{~b}$ ). The initial bands $1 \mathrm{f}, 1 \mathrm{~h}$ are still detectable sometimes. The outline of both these pairs of bands has the same start time of the first wave, 12:50. The appearance of the second pair might be due to the entrance of a part of the shock front into a dense region located rather high above plage A (see Figure 10a,b). The surface EUV wave reached plage A slightly later, as expected for a convex front tilted towards the solar surface (see Section 2.2).

The second shock front was probably manifest in weak bands $2 \mathrm{f}, 2 \mathrm{~h}$ (pale, the start time is 12:56). The bands denoted $(1+2) \mathrm{f}$ and $(1+2) \mathrm{h}$ (orange outline) probably reveal the resulting shock with a virtual start time of 13:01. All of these bands overlap with others increasing the total emission at the intersections. We remind that various bands were most likely emitted from spatially different sites.

We have not revealed manifestations of the two merging shock waves in images or in the kinematical plot. This result could be expected due to different propagation conditions for a shock wave upwards and along the solar surface. The near-surface portion of a shock front decelerates stronger and experiences significant damping, thus becoming weak and approaching a linear disturbance. If the trailing shock wave succeeded to catch up or intersect with the leading one, the effect is expected to approach an interaction of linear disturbances, when two waves pass through each other experiencing interference, while the scheme in Figure 13a shows an essentially nonlinear effect.

The CME was fast $\left(958 \mathrm{~km} \mathrm{~s}^{-1}\right)$ and decelerated. We assume that its leading edge was a trace of a wave and apply a shock-PL fit to the measurements in the CME Catalog at $\mathrm{PA}=270^{\circ}$ (Figure 13b). The onset corresponds to the virtual start time of the coupled shock wave, and the density falloff corresponds to the Saito model. A trailing poorly observed $\mathrm{CME}$ at $\mathrm{PA}=310^{\circ}$ and probably related to the same event was considerably slower $\left(294 \mathrm{~km} \mathrm{~s}^{-1}\right)$. Its deceleration might be due to the influence of the wave running ahead and problems of measurements.

Our analysis does not pretend to be perfect, but it shows that even such a complex dynamic spectrum can be reconciled with EUV observations and CME expansion under assumption of the shock-wave nature of related disturbances. Oversimplified considerations of such a complex event can be misleading. In particular, Yang and Chen (2010) concluded that the EUV wave in this event ran slower in regions of stronger magnetic field that seemed to be a challenge for the wave hypothesis. However, 1) the authors considered the radial component of the magnetic field only, whereas the Alfvén speed depends on its magnitude. 2) By taking the range of the magnetic field strengths $\leq 0.6 \mathrm{G}$, for which Yang and Chen obtained anticorrelation with the EUV wave speed, and a density of $\gtrsim 2 \times 10^{8} \mathrm{~cm}^{-3}$ from the Saito model, one obtains $\beta=(2 / \gamma) C_{\mathrm{s}}^{2} / V_{\mathrm{A}}^{2} \gtrsim 4$, i.e., the wave must be almost insensitive to the magnetic field. 3) The fronts in their Fig. 4 stretch west-southwest and become sharply pointed at 12:59, whereas Long et al. (2008), Veronig, Temmer, and Vršnak (2008), and Attrill (2010) showed the front to be blunt in this direction at that time. 4) Usage of the Huygens plotting to find trajectories of the wave front resulted in a strange picture of intermittently condensed and rarefied ray trajectories in their Fig. 4. Thus, the results of Yang and Chen (2010) do not offer problems for the shock-wave hypothesis. 


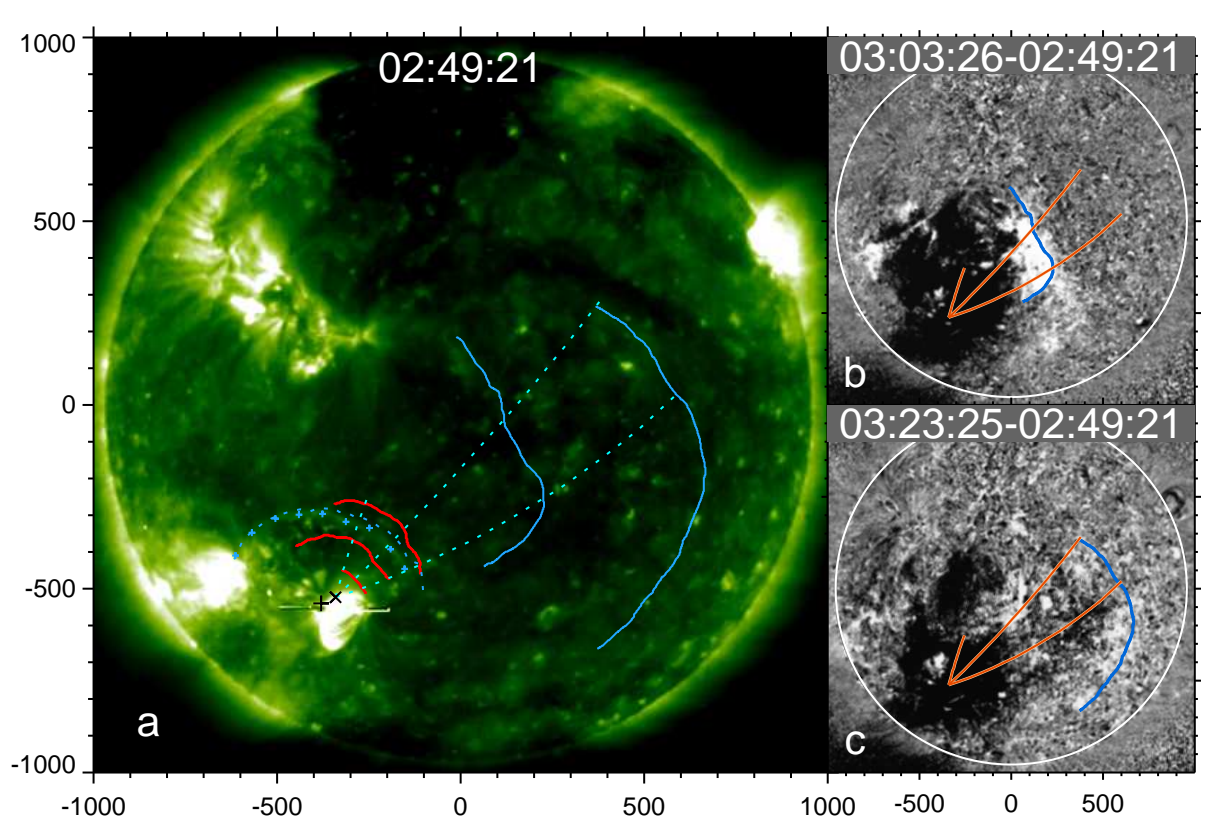

Figure 14. EUV wave (blue) and Moreton wave in the 24 September 1997 event. a) Nonsubtracted EIT $195 \AA$ A image with the first front (blue crosses). Blue dotted line outlines its foremost edge. Red fronts outline the Moreton wave. White broken lines trace the measurement great circles. Black crosses denote the origins of measurements. b,c) Difference ratio images with blue outlines of EUV wave fronts and red measurement great circles. The outlines correspond to Warmuth et al., 2004a (courtesy A. Warmuth).

\subsection{Event 4: 24 September 1997}

This event was associated with a short M5.9 flare (02:43-02:52, S31 E19). An $\mathrm{H} \alpha$ Moreton wave and EUV wave in this event were first analyzed by Thompson et al. (2000). Warmuth et al. (2004a, 2004b) found kinematical closeness of both wave fronts to each other and their deceleration. The first EUV wave front (Figure 14a) was sharp and bright suggesting that the main EUV-emitting layer was low. A difference ratio image (Figure 14b) reveals weak wave manifestations south, southwest, and slightly west from the outline of Warmuth et al. (2004a). The third front in Figure 14c is close to their outline. Deceleration of the EUV wave was therefore even slightly stronger than the authors estimated.

White and Thompson (2005) analyzed wave signatures in microwave images at $17 \mathrm{GHz}$ but did not reveal any deceleration. They also found: $i$ ) the speed of the microwave disturbance was $830 \mathrm{~km} \mathrm{~s}^{-1}$ against $\approx 500 \mathrm{~km} \mathrm{~s}^{-1}$ estimated for the Moreton wave; $i$ ) the brightness temperature at $17 \mathrm{GHz}$ was about five times higher than an estimate from EIT data, and the discrepancy could be reduced if the kinetic temperature at $17 \mathrm{GHz}$ would be different (preferentially higher) from the characteristic temperature of the $195 \AA$ channel. The authors also concluded that the timing of images should be corrected by $\approx 100$ s for EIT and by $\sim 180 \mathrm{~s}$ for $\mathrm{H} \alpha$ to reconcile all observations. These facts indicate that the layers emitting microwaves and EUV were not identical. The higher speed, lesser 

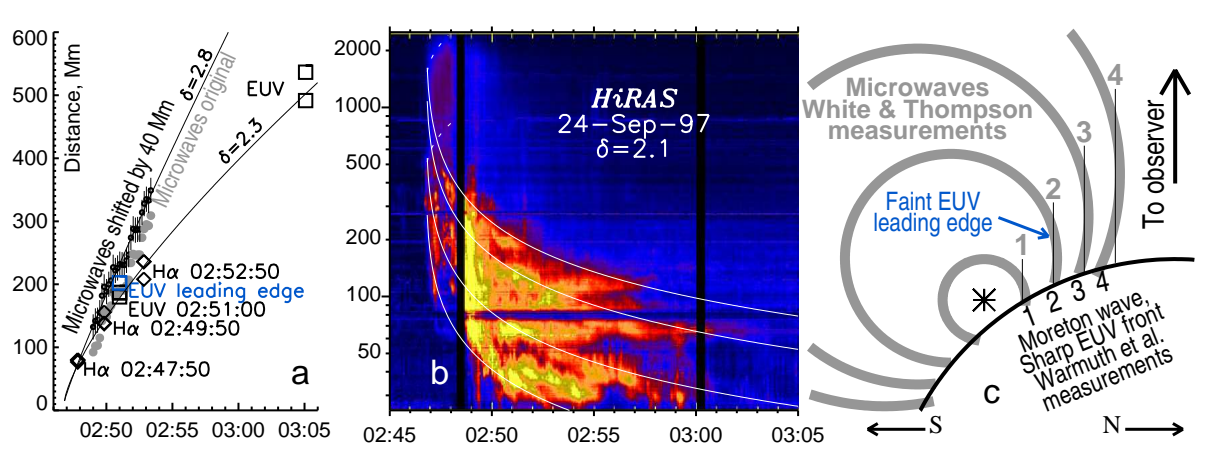

Figure 15. a) Propagation of the EUV wave (squares) and Moreton wave (diamonds) measured by Warmuth et al. (2004a). Filled gray circles show the of White and Thompson (2005) data, black open circles with error bars show them shifted by $40 \mathrm{Mm}$. Both data sets are outlined with shock-PL fit. b) The HiRAS dynamic spectrum outlined with shock-PL fit and a negatively drifting continuum (dotted). c) Presumable relation between the layers emitting microwaves and $\mathrm{EUV} / \mathrm{H} \alpha$. Thin vertical lines show cross sections of the wave fronts of the largest column emission measure contributing at $17 \mathrm{GHz}$. The star is the wave origin.

deceleration, and higher brightness temperature (i.e., column emission measure) observed at $17 \mathrm{GHz}$ with respect to EUV hint at a possibly higher location of the microwave-emitting layer. Figure 15 shows our suggestion in panel (c); panel (a) presents the distance-time plots from both papers. The corrected times of EIT images $(+99 \mathrm{~s})$ and $\mathrm{H} \alpha$ ones $(+170 \mathrm{~s})$ are specified at data points.

To reconcile kinematics of the microwave and $\mathrm{EUV} / \mathrm{H} \alpha$ wave fronts, we shift the White and Thompson data by $40 \mathrm{Mm}$ and fit both data sets with the same onset time of 02:46:50 but different power-law exponents. Figure 15c explains the idea: the lower part of the front propagating in high-density regions decelerated stronger ( $\delta \approx 2.3$ from Warmuth et al. measurements), while microwaves were dominated by long cross sections of the wave front (bars 1-4) running in lowerdensity regions, $\delta \approx 2.8$ ( $c f$. Paper III). The large-height EUV wave's leading edge detectable close to the eruption center diminished at large distances, where the EUV wave was dominated by low structures. The wave presumably appeared at a considerable height (the star). With a difference between the origins of measurements (black crosses in Figure 14a) of $\approx 32 \mathrm{Mm}$, our shift of $40 \mathrm{Mm}$, and spherical wave fronts, we get a height of $117 \mathrm{Mm}$. It seems to be overestimated; an estimate of about $\approx 75 \mathrm{Mm}$ from the dynamic spectrum appears to be more plausible. Thus, the wave could be strongly anisotropic starting from its appearance, or the wave exciter was large, or both.

The complex dynamic spectrum in Figure 15b has a questionable harmonic structure, which is beyond our scope; we are only interested in the drift of the burst envelope. We have outlined presumable fundamental and second-harmonic bands. A possible higher-frequency band and the highest-frequency envelope of the burst are formally outlined as the fourth and sixth harmonic (this does not mean reality of emissions at such high harmonics). The outline corresponds to the initial height of $75 \mathrm{Mm}$, the same wave start time of 02:46:50, and $\delta \approx 2.1$ typical of streamers. A negatively drifting continuum (broken outline) at the initial stage indicates propagation of the shock front towards the chromosphere. 
A poorly observed CME centered at $137^{\circ}$ with a speed of $531 \mathrm{~km} \mathrm{~s}^{-1}$ was injected into a preceding CME. The CME Catalog estimates its acceleration to be positive with a remark about uncertainty. By adding the known origin of the wave (the filled circle in Figure 13c), we get a shock-PL fit of the measurements from the CME Catalog with an exponent $\delta \approx 2.5$ close to the Saito model.

Our considerations confirm correctness of both Warmuth et al. (2004a) and White and Thompson (2005) results, reconcile them with each other and with the type II burst as well as the CME. Deceleration of the front portion detectable at $17 \mathrm{GHz}$ was much less than its lowest part visible in EUV and $\mathrm{H} \alpha$ had. It was not possible to reveal deceleration from microwave observations, which allowed detection of the wave within an interval as short as $4.5 \mathrm{~min}$.

\section{Discussion}

TRACE observations of abrupt eruptions in events 1 and 2 have revealed planeof-sky accelerations of magnetic rope structures of $4-7 \mathrm{~km} \mathrm{~s}^{-2}$, i.e., $(15-25)$-fold gravity acceleration. Then the eruptions in both events decelerated. Coronal waves appeared in events $1-3$ approximately at the peak of acceleration. The onsets of the waves in all four events corresponded to the rise phases of HXR or microwave bursts (in event 4 according to White and Thompson, 2005). The wave in event 1 steepened into a shock within one minute and reached a Mach number of about 1.3 in a horizontal direction, while the upwards shock could be stronger. In the next Section we consider which of known shock formation scenarios (see, e.g., Vršnak and Cliver, 2008) appear to match the observations.

\subsection{Comparison of Observations with Shock Formation Scenarios}

A scenario of the shock formation by a flare pressure pulse is based on an idea that the $\beta$ ratio of the plasma pressure to the magnetic pressure sharply changes from $\beta \ll 1$ to $\beta>1$. Such a change of $\beta$ is believed to be possible in a flare loop and considered as a cause of an omnidirectional disturbance. Dramatic changes of the volume of a loop or its abrupt motion are necessary to get a significant

intensity of a wave excited in this way. Objections against this scenario do exist.

1) As Grechnev et al. (2006c) showed, the effect of a high $\beta$ (even $\beta>1$ ) in a flare loop is not dramatic, only an increase of all its sizes of $\sqrt[4]{1+\beta}$. 2) The idea that a situation of $\beta \rightarrow 1$ can cause instability of a loop is not confirmed by observations. Ichimoto et al. (1993) and Grechnev et al. (2006a) showed that the $\beta<1$ condition was not satisfied in long-lived hot coronal loops. 3) The flare pressures in events 1 and 2 rose gradually, without any marks of the wave appearance. 4) RHESSI showed the SXR-emitting regions in events 1 and 2 to be fixed when the pressure increased. Veronig, Temmer, and Vršnak (2008) concluded that the wave ignition in event 3 by the flare was unlikely. Thus, the theoretical considerations supported by the observational facts make the ignition of waves by flares doubtful in agreement with the conclusion of Cliver et al. (2004) (note that the authors implied the shocks to be driven by outer surfaces of CMEs, whereas we consider the shocks to appear inside CMEs). 
This conclusion is seemingly opposed by the results of Magdalenić et al. $(2008,2010)$ who presented five events, in which shocks appeared during the flare impulsive phase, whereas related CMEs were slow. However, the authors did not consider a rare type of CME kinematics with very strong initial acceleration followed by deceleration. An example is our event 1 (Section 3.1, Figure 4). The acceleration stage in this event lasted three minutes. The fastest feature accelerated up to $470 \mathrm{~km} \mathrm{~s}^{-1}$ and continuously decelerated later on. From an overall height - time plot, including measurements from LASCO images, Grechnev et al. (2008) estimated the speed of this feature to approach $100 \mathrm{~km} \mathrm{~s}^{-1}$ far from the

Sun. Nevertheless, the shock in this event was most likely excited just by this feature as an impulsive piston rather than by the flare. Thus, a slower motion of the main body of a CME well behind a shock front does not guarantee that the CME or its components were not implicated in excitation of the shock.

The maximum plane-of-sky speeds of the eruptions in events $1-3$ appear to be well below the Alfvén speed expected at moderate heights $(<100 \mathrm{Mm})$ above active regions (e.g., Mann et al., 2003), where the waves appeared. It is possible that the angles between the velocity vectors and the line of sight significantly differed from $90^{\circ}$, so that the real velocities could be higher, but the corresponding corrections are insufficient to increase the velocities above the Alfvén speed. The time profiles of the velocities estimated for the eruptions and waves were quite different. Thus, the bow shock scenario is also unlikely.

\subsection{Impulsive Piston Scenario}

In a simplest scheme, a piston moving with a speed $U$ has a box-like acceleration profile with a value $a$ during the acceleration phase and zero before and afterwards. An important condition is that plasma cannot flow around the piston (this occurs, e.g., in 3D expansion of an arcade). In a flat geometry and homogeneous medium the plasma flow ahead of the piston corresponds to a simple wave. The discontinuity appears at $t_{\mathrm{sh}}^{0}=V_{\text {fast }} /(\kappa a)$ at a distance $r_{\mathrm{sh}}^{0}=V_{\text {fast }} t_{\mathrm{sh}}^{0}$ with $1 / 2 \leq \kappa \leq 3 / 2$ that is similar to a solution of an analogous gas-dynamic problem (Landau and Lifshits, 1987). Then the speed jump in the discontinuity increases up to the piston's maximum speed $U_{\max }$. The condition $U>V_{\text {fast }}$ essential for bow shock formation is unnecessary in the impulsive piston scenario.

Accelerations and their durations before the wave onsets were (Section 3): $4 \mathrm{~km} \mathrm{~s}^{-2}$ and $90 \mathrm{~s}$ in event $1 ; 7 \mathrm{~km} \mathrm{~s}^{-2}$ and $70 \mathrm{~s}$ in event $2 ; 0.12-0.27 \mathrm{~km} \mathrm{~s}^{-2}$ and $800 \mathrm{~s}$ in event 3 . The stronger acceleration, the faster a shock appeared.

The shock waves in the four events were most likely excited by eruptive structures as impulsive pistons, which one might call the appearing CMEs. Then the waves rapidly steepened into shocks, detached the pistons, and freely propagated afterwards like blast waves. The shock excitation mechanism implies a source height to be nonzero, but rather low, probably $<100 \mathrm{Mm}$, as suggested by all dynamic spectra and implied by event 4 . Magdalenić et al. $(2008,2010)$ found the heights of sources of metric type II bursts to be between 70 and $280 \mathrm{Mm}$; however, the apparent heights of limb sources in the metric range could be noticeably reduced due to refraction (see, e.g., Zheleznyakov, 1970). The shock character of the waves is supported by the correspondence of their kinematics 
to the expected propagation of shock waves as well as the drift rates of type II bursts and drifting continua. The shock-wave nature of EUV waves is supported with probable reflections and coupling of two shock waves in event 3. Expansion of leading edges of CMEs produced in three events corresponded to propagation of the lower skirts of the shock fronts observed as Moreton/EUV waves. Thus, the wave excitation by an impulsive piston appears to match all the considered observations, basically corresponding to a picture proposed by Uchida (1974).

So far we did not relate a piston with a particular structure or its surface. Presumable pistons could be either an eruptive filament (EF) or a CME frontal structure (FS). Both expand as an entire ensemble in a completely formed CME; only its outer sheath can be a piston. This sheath is believed to be both the surface of contact discontinuity and the outer FS surface. Expansion of such an FS-piston determines propagation of an interplanetary piston-driven wave and the drag force affecting a CME. The situation is different during the early CME formation inside an active region, when EF moves faster than it would be adequate for a self-similar expansion of the whole CME. The EF acts here as an impulsive piston and excites inside a future CME a wave, which freely propagates outwards as a shock wave. In the four events we revealed just this excitation scenario of waves, which resembled blast ones. Propagating upwards, such a wave inevitably would pass through the FS and appear ahead of it.

\subsection{CME Components and Waves}

\subsubsection{Particularities of Expansion}

Expansion of a magnetoplasma CME's constituent is different from kinematics of a wave traced, e.g., with a leading edge of a plasma flow driven by a shock. The CME expansion is known to be about self-similar at moderate distances from the Sun. The self-similar approach does not apply to early stages of expansion, when the structure and shape of a CME have not yet been established. When an instability driving an eruption completes and drag of the solar wind is not yet significant, the self-similar CME kinematics can be obtained from considerations of forces affecting a CME (Low, 1982; Uralov, Grechnev, and Hudson, 2005).

Expansion of magnetoplasma structures is governed by magnetic forces, plasma pressure, and gravity as long as the effect of the solar wind is small. With the polytropic index $\gamma \approx 4 / 3$ all the forces integrated over the boundary and volume of a CME scale with distance $r$ by the same factor of $r^{-2}$. This leads to an expression for the CME velocity $V_{\mathrm{CME}}^{2}=V_{0}^{2}+\left(V_{\infty}^{2}-V_{0}^{2}\right)\left(1-R_{0} / r\right), V_{\mathrm{CME}}=d r / d t$ (Grechnev et al., 2008). Here $R_{0}$ is the initial size of self-similar expansion, $V_{0}$ the initial velocity at $R_{0}$, and $V_{\infty}$ the asymptotic velocity in infinity. At large distances acceleration $\propto r^{-2} \rightarrow 0$ and $V_{\mathrm{CME}} \rightarrow V_{\infty}$. The expression for the CME velocity describes different types of kinematics. The situation $V_{\infty} \gg V_{0} \sim 0$ appears to be typical. Event 1 showed a different behavior, $V_{0}>V_{\infty}$, resembling an explosion with an impulsive acceleration followed by deceleration. A special type $V_{0} \approx V_{\infty}$ (an impulsive acceleration is required to reach $V_{0}$ ) might correspond

to some jet-like ejections, where magnetic reconnection destroys a structure of an eruption (Meshalkina et al., 2009; Filippov, Golub, and Koutchmy, 2009; Liu et al., 2011). Event 2 might have belonged to this type. 
Since a FS starts to expand practically from a static equilibrium in a typical situation $V_{\infty} \gg V_{0} \sim 0$, the FS-piston usually either accelerates or moves with a nearly constant speed at the self-similar stage. By contrast, shock waves in all considered events decelerated. Hence, a typical FS-piston is expected to eventually approach the wave front. What does such a relation between the speeds of the shock front and piston mean? Do MHD equations allow a decelerating shock wave to run for a long time ahead of a non-decelerating FS-piston? We search answers in a theory developed by Low (1984) in solving a problem of self-similar expansion of a CME preceded by a strong shock wave. Though the problem was solved in a limit of very strong shock propagating in plasma with too steep density falloff $r^{-26 / 7}$, the solution correctly relates accelerations of the piston and piston-driven shock. Assuming a common linear profile of the plasma velocity in the whole region from the expansion center up to the shock front, it is possible to relate kinematics of the shock front and the contact discontinuity, i.e., the FS-piston without a complete solution of the problem. By fitting the motion of the piston with a function $r_{\text {pist }}=b t^{m}$, we express the sign of the shock acceleration $a_{\text {sh }}$ vs. $m: a_{\text {sh }} \propto(\alpha m-1) t^{\alpha m-2}$ with $\alpha=(\gamma+1) / 2$. Thus, the conditions $a_{\mathrm{sh}}<0$ and $a_{\text {pist }}=d^{2} r_{\text {pist }} / d t^{2}>0$ are incompatible. Such an FSpiston and the shock front expand in different ways, which cannot be coordinated with each other, unlike a bow shock. An impulsively excited freely propagating shock wave must eventually change to a piston-driven mode. Presumably this typically occurs at large distances, probably beyond the LASCO/C3 field of view. The transformation of a blast shock wave into a piston-driven one marks switchon the aerodynamic drag and termination of the self-similar expansion regime. The drag force becomes significant, which means establishment of a continuous energy transport from the FS-piston to the shock wave. By contrast, a blast-like wave excited by an EF and running ahead of FS, which does not yet act as a piston, facilitates expansion of a CME into the solar wind. The shock wave forwards a part of its energy to the FS-piston, and the drag force is absent.

Most likely, real shock waves are neither purely blast waves nor purely piston ones. A shock front is sensitive to any events occurring behind it, e.g., changes of the FS-piston speed, because the fast-mode speed behind the shock front is higher than its phase speed. To produce one more shock wave, an FS-piston has to repeat the maneuver, which produced the first shock. This is improbable when an instability driving an eruption has completed and a CME left the Sun.

\subsubsection{Distinguishing between Shock Signatures and CME Components}

Sheeley, Hakala, and Wang (2000) and Vourlidas et al. (2003) considered distortions of coronal streamers as a morphological suggestion of presumable shocks. Indeed, moving wave-like kinks or deflections of coronal rays resemble an expected effect of a propagating shock wave. Some fast "CMEs" in difference images might be actually combinations of coronal rays deflected by shock waves. However, Filippov and Srivastava (2010) demonstrated that deflection of coronal rays could be due to expansion of a CME in magnetized corona without a shock. Irrespective of a particular type of a possible shock, more reliable morphological suggestion might be a spike-like leading feature due to deviation of a coronal 
ray by a wave. Magdalenić et al. (2008) showed such a situation in their Fig. 4, where deflected coronal rays were visible well ahead of trailing CME structures.

We remind that the conic bow-shock shape is not expected, at least, for wide super-Alfvénic CMEs. The shock front must cling to its foremost edge and closest flanks, while far flanks and a rear part can be constituted by a freely propagating shock front, so that the shape of the whole front would resemble an egg.

An attractive way to detect a shock front is to search for discontinuity in the density distribution shown by coronagraph images (Vourlidas et al., 2003). However, this way is model dependent. Besides modeling the coronal density, one has to distinguish between the shock discontinuity and the contact surface separating the CME and environment. The 3-dimensional contact surface should be also modeled. Thus, identifying a shock front in coronagraph images does not seem to be a simple task. Combinations of different indications seem to be be useful. A non-structured faint density enhancement forming the envelope of a transient could be among them (Vourlidas et al., 2003). Fast decelerating halo CMEs with such edges might be shock candidates. A worthwhile shock indication might be such a halo edge crossing a distorted streamer. An important complement of morphological suggestions is kinematics of an expected shock wave and its correspondence to the drift of a type II burst. Paper III also address changes in shape of a shock front occurring in its propagation in the corona.

\subsection{Presumable Scenario}

Our observations and considerations suggest the following presumable scenario of a flare-related eruptive event. An eruption occurs due to a rapid development of an instability in a magnetic structure. An abruptly accelerating eruption destroys a pre-existing magnetic configuration, thus causing a flare, and produces an MHD disturbance as an impulsive piston. The disturbance appears at a height of $\sim 50 \mathrm{Mm}$ during the rise phase of an HXR/microwave burst, leaves the piston, rapidly steepens into a shock, and then freely propagates like a blast wave. Its lower trail might be observed as a decelerating Moreton wave as well as an EUV wave, and the wave dome is sometimes observed to expand above the limb.

The motion of the shock front shows up in radio spectra as a drifting continuum and, when the shock front reaches the current sheet of a coronal streamer, as a type II burst. For the fundamental emission this usually occurs at $\sim 100$ $\mathrm{MHz}\left(r \sim 1.5 R_{\odot}\right)$. Metric type II bursts are expected to cease due to damping of shock waves that typically occurs above $20 \mathrm{MHz}\left(r<3 R_{\odot}\right)$. Revival of a shock is possible at a few $R_{\odot}$ due to decreasing Alfvén speed, and decametric/hectometric type II emission can appear. The complex piston-blast-piston transformations of shock waves traveling in the corona with significantly varying parameters and possible coupling of multiple shocks imply well-known disagreement between metric and interplanetary type II events (e.g., Cane and Erickson, 2005).

Expanding shock fronts can form envelopes of CMEs. Measurements in the CME Catalog referring to a fastest feature might be related to shock waves for fast decelerating CMEs, especially halos. Since a shock wave decelerates, a trailing mass must eventually approach its front. The shock becomes a pistondriven one presumably at distances $r>20 R_{\odot}$. The aerodynamic drag becomes 
important. This picture is consistent with results of several cited papers. The story of shock waves associated with flare-related CMEs appears to be more complex than often assumed, in fact combining different scenarios.

\section{Concluding remarks}

Our seemingly simplified approach has resulted in surprisingly fine reconciliation of EUV waves, Moreton waves, metric type II bursts, and leading edges of CMEs. The first consequence is that independent of the quality of our approximation, all these phenomena are really manifestations of a common agent, i.e., a traveling coronal shock wave excited by an eruption. Second, our approach indeed provides a promising instrument for analyses slow-drifting bursts and their comparison with other eruption-related phenomena. The power-law approximation turns out to work well beyond conditions, for which it was derived. Our results clarify relations between flares, traveling coronal shocks, CMEs, associated wave-like manifestations, type II bursts, and provide a common quantitative description for some of these phenomena. Our important by-product is an indication of the leading role of eruptions with respect to flares, i.e., that the acceleration of an eruption occurs almost independently of the flare reconnection rate.

Our approach and analysis needs elaboration and continuation. A number of issues to be addressed remains. Low heights, at which type II emission sometimes appears, indicate that our consideration of its generation in the current sheet of a coronal streamer needs elaboration. Data sets similar to those analyzed in our paper should be compared with imaging observations in the metric range. The analysis of decimetric to metric drifting bursts should be extended to longer radio waves in conjunction with coronagraphic observations. Despite success of our self-similar shock approximation, a more realistic weak shock approximation should be considered. The last issue is a subject of our paper II.

Acknowledgements We thank A. Warmuth for data, which he made available to us, and M. Eselevich, E. Ivanov, E. Schmahl, M. Temmer, V. Eselevich, A. Altyntsev, G. Rudenko, L. Kashapova, V. Fainshtein, N. Prestage, S. Pohjolainen, S. White, A. Zhukov, and J. Magdalenić for fruitful discussions and cooperation. We gratefully remember Mukul Kundu who inspired a significant part of our study. We thank an anonymous referee for valuable remarks.

We thank the teams operating all instruments whose data are used in our study for their efforts and open data policies: the ESA \& NASA EIT, LASCO, and MDI instruments on SOHO; TRACE and STEREO/SECCHI telescopes; the Mauna Loa Solar Observatory; the NOAA/SEC GOES satellites; the NICT HIRAS (Japan), the IPS Radio and Space Services Learmonth Observatory (Australia), and the USAF RSTN radio telescopes. We appreciatively use the CME catalog generated and maintained at the CDAW Data Center by NASA and the Catholic University of America in cooperation with the Naval Research Laboratory. This research was supported by the Russian Foundation of Basic Research under grant 09-02-00115.

\section{References}

Afanasyev, A. N., Uralov, A. M.: 2011, Solar Phys. In this issue, DOI: 10.1007/s11207-0119730-9.

Attrill, G. D. R.: 2010, Astrophys. J. 718, 494.

Biesecker, D. A., Myers, D. C., Thompson, B. J., Hammer, D. M., Vourlidas, A.: 2002, Astrophys. J. 569, 1009. 
Brueckner, G. E, Howard, R. A., Koomen, M. J., Korendyke, C. M., Michels, D. J., Moses, J. D., et al.: 1995, Solar Phys. 162, 357.

Cane, H. V., Erickson, W. C.: 2005, Astrophys. J. 623, 1180.

Chen, P. F., Fang, C., Shibata, K.: 2005, Astrophys. J. 622, 1202.

Chertok, I. M., Grechnev, V. V.: 2005, Solar Phys. 229, 95.

Chertok, I. M., Grechnev, V. V., Uralov, A. M.: 2009, Astron. Rep. 53, 355.

Cliver, E. W., Nitta, N. V., Thompson, B. J., Zhang, J.: 2004, Solar Phys. 225, 105.

Cohen, O., Attrill, G. D. R., Manchester, W. B., Wills-Davey, M. J.: 2009, Astrophys. J. 705, 587.

Delaboudinière, J.-P., Artzner, G. E., Brunaud, J., Gabriel, A. H., Hochedez, J. F., Millier, F. et al.: 1995, Solar Phys. 162, 291.

Delannée, C.: 2000, Astrophys. J. 545, 512.

Filippov, B., Golub, L., Koutchmy, S.: 2009, Solar Phys. 254, 259.

Filippov, B., Srivastava, A. K.: 2010, Solar Phys. 266, 123.

Gallagher, P. T., Long, D. M.: 2010, Space Sci. Rev. 127.

Gopalswamy, N., Yashiro, S., Temmer, M., Davila, J., Thompson, W. T., Jones, S., McAteer, R. T. J., Wuelser, J.-P., Freeland, S., Howard, R. A.: 2009, Astrophys. J. Lett. 691, 123.

Grechnev, V. V., Afanasyev, A. N., Uralov, A. M., Chertok, I. M., Eselevich, M. V., Eselevich, V. G., Rudenko, G. V., Kubo, Y.: 2011, Solar Phys. In this issue.

Grechnev, V. V., Kuzin, S. V., Urnov, A. M., Zhitnik, I. A., Uralov, A. M., Bogachev, S. A., Livshits, M. A., Bugaenko, O. I. et al.: 2006a, Sol. Sys. Res. 40, 286.

Grechnev, V. V., Uralov, A. M., Slemzin, V. A., Chertok, I. M., Kuzmenko, I. V., Shibasaki, K.: 2008, Solar Phys. 253, 263.

Grechnev, V. V., Uralov, A. M., Zandanov, V. G.; Baranov, N. Y.; Shibasaki, K.: 2006b, Pub. Astron. Soc. Japan 58, 69.

Grechnev, V. V., Uralov, A. M., Zandanov, V. G., Rudenko, G. V., Borovik, V. N., Grigorieva, I. Y., Slemzin, V. A., Bogachev, S. A. et al.: 2006c, Pub. Astron. Soc. Japan 58, 55.

Handy, B. N., Acton, L. W., Kankelborg, C. C., Wolfson, C. J., Akin, D. J., Bruner, M. E., Caravalho, R., Catura, R. C., et al.: 1999, Solar Phys. 187, 229.

Howard, R. A., Moses, J. D., Vourlidas, A., Newmark, J. S., Socker, D. G., Plunkett, S. P., Korendyke, C. M., Cook, J. W. et al.: 2008, Space Sci. Rev. 136, 67.

Hudson, H. S., Khan, J. I., Lemen, J. R., Nitta, N. V., Uchida, Y.: 2003, Solar Phys. 212, 121.

Hudson, H. S., Warmuth, A.: 2004, Astrophys. J. Lett. 614, 85.

Ichimoto, K., Sakurai, T., Flare Telescope, Norikura Teams: 1993, Proc. 2nd Japan-China seminar on Sol. Phys., p. 151.

Kaiser, M. L., Kucera, T. A., Davila, J. M., St. Cyr, O. C., Guhathakurta, M., Christian, E.: 2008, Space Sci. Rev. 136, 5.

Khan, J. I., Aurass, H.: 2002, Astron. Astrophys. 383, 1018.

Klassen, A., Aurass, H., Mann, G., Thompson, B. J.: 2000, Astron. Astrophys. Suppl. 141, 357.

Landau, L. D., Lifshitz, E. M.: 1987, Fluid Mechanics, 2nd edn., Oxford, Pergamon Press.

Lin, R. P., Dennis, B. R., Hurford, G. J., Smith, D. M., Zehnder, A., Harvey, P. R., et al.: 2002, Solar Phys. 210, 3.

Liu, W., Berger, T. E., Title, A. M., Tarbell, T. D., Low, B.C.: 2011, Astrophys. J. 728, article id. 103.

Long, D. M., Gallagher, P. T., McAteer, R. T. J.. Bloomfield, D. S.: 2008, Astrophys. J. Lett. $680,81$.

Low, B. C.: 1982, Astrophys. J. 254, 796.

Low, B. C.: 1984, Astrophys. J. 281, 381.

Magdalenić, J., Marqué, C., Zhukov, A. N., Vršnak, B., Žic, T.: 2010, Astrophys. J. 718, 266.

Magdalenić, J., Vršnak, B., Pohjolainen, S., Temmer, M., Aurass, H., Lehtinen, N. J.: 2008, Solar Phys. 253, 305.

Mancuso, S., Abbo, L.: 2004, Astron. Astrophys. 415, L17.

Mancuso, S., Raymond, J. C.: 2004, Astron. Astrophys. 413, 363.

Mann, G., Klassen, A., Aurass, H., Classen, H.-T.: 2003, Astron. Astrophys. 400, 329.

Meshalkina, N. S., Uralov, A. M., Grechnev, V. V., Altyntsev, A. T., Kashapova, L. K.: 2009, Pub. Astron. Soc. Japan 61, 791.

Moreton, G. E.: 1960, Astronom. J. 65, 494.

Nakajima, H., Sekiguchi, H., Sawa, M., Kai, K., Kawashima, S.: 1985, Pub. Astron. Soc. Japan 37, 163.

Newkirk, G. Jr.: 1961, Astrophys. J. 133, 983. 
Pohjolainen, S., Hori, K., Sakurai, T.: 2008, Solar Phys. 253, 291.

Pomoell, J., Vainio, R., Kissmann, R.: 2008, Solar Phys. 253, 249.

Reiner, M. J., Vourlidas, A., St. Cyr, O. C., Burkepile, J. T., Howard, R. A., Kaiser, M. L., Prestage, N. P., Bougeret, J.-L.: 2003, Astrophys. J. 590, 533.

Rudenko, G. V.: 2001, Solar Phys. 198, 5.

Rudenko, G. V., Grechnev, V. V.: 1999, Astronomical Data Analysis Software and Systems VIII, ASP Conf. Series, 172, 421.

Saito, K.: 1970, Ann. Tokyo Astr. Obs. 12, 53.

Schmidt, J. M., Ofman, L.: 2010, Astrophys. J. 713, 1008.

Sheeley, N. R., Jr., Hakala, W. N., Wang, Y.-M.: 2000, J. Geophys. Res. 105, A3, 5081.

Temmer, M., Veronig, A. M., Vršnak, Rybák, J., Gömöry, J., Stoiser, S., Maričić, D.: 2008, Astrophys. J. Lett. 673, 95.

Thompson, B. J., Gurman, J. B., Neupert, W. M., Newmark, J. S., Delaboudinière, J.-P., St. Cyr, O. C., Stezelberger, S., Dere, K. P.: 1999, Astrophys. J. Lett. 517, 151.

Thompson, B. J., Plunkett, S. P., Gurman, J. B., Newmark, J. S., St. Cyr, O. C., Michels, D. J.: 1998, Geophys. Res. Lett. 25, 2465.

Thompson, B. J., Reynolds, B., Aurass, H., Gopalswamy, N., Gurman, J. B., Hudson, H. S., Martin, S. F., St. Cyr, O. C.: 2000, Solar Phys. 193, 161.

Torii, C., Tsukiji, Y., Kobayashi, S., Yoshimi, N., Tanaka, H., Enome, S.: 1979, Proc. Res. Inst. Atmospherics 26, 129.

Uchida, Y.: 1968, Solar Phys. 4, 30.

Uchida, Y.: 1974, Solar Phys. 39, 431.

Uralov, A. M., Grechnev, V. V., Hudson, H. S.: 2005, J. Geophys. Res. 110, A05104.

Uralova, S. V., Uralov, A. M.: 1994, Solar Phys. 152, 457.

Veronig, A. M., Muhr, N., Kienreich, I. W., Temmer, M., Vršnak, B.: 2010, Astrophys. J. Lett. 716, 57.

Veronig, A. M., Temmer, M., Vršnak, B.: 2008, Astrophys. J. Lett. 681, 113.

Vourlidas, A., Wu, S. T., Wang, A. H., Subramanian, P., Howard, R. A.: 2003, Astrophys. J. 598, 1392.

Vršnak, B., Aurass, H., Magdalenić, J., Gopalswamy, N.: 2001, Astron. Astrophys. 377, 321.

Vršnak, B., Cliver, E. W.: 2008, Solar Phys. 253, 215.

Wang, Y., Zhang, J., Shen, C.: 2009, J. Geophys. Res. 114, 10104.

Warmuth, A.: 2007, Lecture Notes in Physics, Berlin Springer Verlag 725, 107.

Warmuth, A.: 2010, Adv. Space Res. 45, 527.

Warmuth, A., Vršnak, B., Aurass, H., Hanslmeier, A.: 2001, Astrophys. J. Lett. 560, 105.

Warmuth, A., Vršnak, B., Magdalenić, J., Hanslmeier, A., Otruba, W.: 2004a, Astron. Astrophys. 418, 1101.

Warmuth, A., Vršnak, B., Magdalenić, J., Hanslmeier, A., Otruba, W.: 2004b, Astron. Astrophys. 418, 1117.

Wills-Davey, M. J., Attrill, G. D. R.: 2009, Space Sci. Rev. 149, 325.

Wills-Davey, M. J., DeForest, C. E., Stenflo, J. O.: 2007, Astrophys. J. 664, 556.

White, S. M., Thompson, B. J.: 2005, Astrophys. J. Lett. 620, 63.

Yang, H. Q., Chen, P. F. 2010, Solar Phys. 266, 59.

Yashiro, S., Gopalswamy, N., Michalek, G., St. Cyr, O. C., Plunkett, S. P., Rich, N. B., Howard, R. A.: 2004, J. Geophys. Res. 109, A07105.

Zheleznyakov, V. V.: 1970, Radio Emission of the Sun and the Planets, Pergamon Press Ltd., Oxford.

Zhukov, A. N., Auchère, F.: 2004, Astron. Astrophys. 427, 705.

Zhukov, A. N., Rodriguez, L., de Patoul, J.: 2009, Solar Phys. 259, 73. 